\title{
On the GBDT Version of the Bäcklund-Darboux Transformation and its Applications to Linear and Nonlinear Equations and Weyl Theory
}

\author{
A. Sakhnovich * \\ Department of Mathematics, University of Vienna, Nordbergstrasse 15, A-1090 Vienna, Austria
}

To the memory of M.Sh. Birman, with deep respect

\begin{abstract}
A general theorem on the GBDT version of the Bäcklund-Darboux transformation for systems depending rationally on the spectral parameter is treated and its applications to nonlinear equations are given. Explicit solutions of direct and inverse problems for Dirac-type systems, including systems with singularities, and for the system auxiliary to the $N$-wave equation are reviewed. New results on explicit construction of the wave functions for radial Dirac equation are obtained.
\end{abstract}

Key words: Bäcklund-Darboux transformation, Weyl function, reflection coefficient, direct problem, inverse problem, Dirac-type system, radial Dirac equation, integrable equation.

AMS subject classification: 37K35, 34B20, 47A48, 37K10, 47A40

\section{Introduction}

Bäcklund-Darboux transformations (BDTs) are well-known as a versatile tool in spectral theory as well as for integrable nonlinear equations (see, for instance, $[3,7,9,13,14,15,19,25,34,42,43$, $44,47,49,51,85]$ and references therein). BDT transforms initial equation or system into another

*E-mail: al_sakhnov@yahoo.com 
one from the same class and transforms also solutions of the initial equation into solutions of the transformed one.

In this paper we review results on the generalized Bäcklund-Darboux transformation (GBDT), where $n \times n$ matrices are used as generalized eigenvalues (see explanations regarding matrix functions $\Pi_{k}(x)$ further in the introduction as well as Remark 2.4 and formulas (3.3) and (3.4) for details). The GBDT version of the Bäcklund-Darboux transformation works for the matrix and scalar cases, gives explicit expression for the iterated Darboux matrix in terms of a transfer matrix function, minimizes the order of the matrix that has to be inverted in the BDT approach. Thus, GBDT is a convenient tool to construct wave functions and explicit solutions of the nonlinear wave equations as well as to solve various direct and inverse problems. GBDT and its applications were treated or included as important examples in the papers $[22,23,48,55,56,57,58,59,61$, $63,64,66,67,68,70,71,72,73,75]$ (see also [28, 29, 30, 31, 32, 33, 37]). Here we consider self-adjoint and skew-self-adjoint Dirac-type systems including the singular case corresponding to soliton-positon interaction and solve direct and inverse problems. We solve also direct and inverse problems for the system auxiliary to the $N$-wave equation, construct explicit solutions of the $N$ wave equation and obtain evolution of the corresponding Weyl function. The results on the radial Dirac equation are new and we treat them in some detail. We consider also in a detailed way a general Theorem 3.1 on the GBDT for systems depending rationally on the spectral parameter and its applications. (This result was earlier published in [57].)

First, let us illustrate BDT by the oldest and most popular example, that is, by the SturmLiouville equation

$$
-\frac{d^{2}}{d x^{2}} y(x, \lambda)+v(x) y(x, \lambda)=\lambda y(x, \lambda),
$$

where $v(x)=\bar{v}(x), \lambda=\bar{\lambda}$. Assume that $z(x)=\bar{z}(x)$ satisfies (1.1), when $\lambda=c$, that is, $-z_{x x}+v z=c z\left(z_{x x}:=\frac{d^{2}}{d x^{2}} z\right)$. Then one can rewrite (1.1) in the form

$$
\left(\mathcal{A}^{*} \mathcal{A}+c I\right) y(x, \lambda)=\lambda y(x, \lambda)
$$

where $\mathcal{A}$ and $\mathcal{A}^{*}$ are first order differential expressions:

$$
\mathcal{A} f=\left(\frac{d}{d x}-\frac{z_{x}}{z}\right) f, \quad \mathcal{A}^{*} f=-\left(\frac{d}{d x}+\frac{z_{x}}{z}\right) f .
$$

Transformed equation is given by the formula

$$
\left(\mathcal{A} \mathcal{A}^{*}+c I\right) y(x, \lambda)=\lambda y(x, \lambda)
$$

It easy to see that (1.3) is again Sturm-Liouville equation, but potential $v$ is transformed into $\widetilde{v}=v-2\left(\frac{z_{x}}{z}\right)_{x}$. Notice further that $\left(\mathcal{A} \mathcal{A}^{*}+(c-\lambda) I\right) \mathcal{A}=\mathcal{A}\left(\mathcal{A}^{*} \mathcal{A}+(c-\lambda) I\right)$. Hence, it follows that if $y(x, \lambda)$ satisfies (1.2), then $\widetilde{y}:=\mathcal{A} y$ satisfies (1.3). Fundamental solutions of the transformed equations can be constructed in this way. Under rather weak conditions the spectra of operators $L^{*} L$ and $L L^{*}$ may differ only at zero, and so under certain conditions the spectra of Sturm-Liouville operators $\mathcal{L}$ and $\widetilde{\mathcal{L}}$ associated with differential expressions $-\frac{d^{2}}{d x^{2}}+v$ and $-\frac{d^{2}}{d x^{2}}+\widetilde{v}$ 
may differ only at $c$. The Bäcklund-Darboux (and related commutation) methods of inserting and removing eigenvalues of Sturm-Liouville operators historically go back to Bäcklund, Darboux, and Jacobi [7, 19, 35] with decisive later contributions by Crum, Deift, and Gesztesy [18, 20, 24]. (See also [26, 27] and a detailed account in Appendix G in [25].)

One can apply BDT again to the already transformed equation (1.3) and so on (iterated BDT). There is also somewhat more complicated binary BDT (see [1, 47]). It proves that, if $v$ satisfies nonlinear integrable equation, then $\widetilde{v}$ often satisfies it too, and so BDT is used to construct solutions of the nonlinear equations. BDT proves especially useful for the construction of the explicit solutions, starting from the trivial initial system (for instance, $v=0$ in (1.1)).

Elementary Bäcklund-Darboux transformations for Dirac type and more general AKNS systems one can find, for instance, in [13,41]. Given first order initial and transformed systems $u_{x}=G(x, \lambda) u$ and $\widetilde{u}_{x}=\widetilde{G}(x, \lambda) \widetilde{u}$, their solutions are connected via so called Darboux matrix $w$ such that $w_{x}=\widetilde{G} w-w G$. Here $G, \widetilde{G}$ and $w$ are $m \times m$ matrix functions $(m>0)$. Clearly, if $u$ satisfies some initial system $u_{x}=G u$, then $w u$ satisfies the transformed one $\widetilde{u}_{x}=\widetilde{G} \widetilde{u}$. Darboux matrix or gauge transformation is of great interest in this theory (see $[15,41,43,47,52,79,81]$ and references therein).

We assume that (if it is not stated otherwise) the fundamental solutions $u$ of the considered systems are normalized by the condition

$$
u(0, \lambda)=I_{m}
$$

where $I_{m}$ is the $m \times m$ identity matrix. Then the fundamental solution $\widetilde{u}$ of the transformed system (normalized by $\left.\widetilde{u}(0, \lambda)=I_{m}\right)$ is given by the formula $\widetilde{u}(x, \lambda)=w(x, \lambda) u(x, \lambda) w(0, \lambda)^{-1}$, where $u$ is the fundamental solution of the initial system.

We shall consider the GBDT version of the Bäcklund-Darboux transformation, where the Darboux matrix is represented in the form of the transfer matrix function $w(x, \lambda)=w_{A}(x, \lambda)$. Transfer matrix function corresponding to the $S$-node (transfer matrix function in the L. Sakhnovich form) is given by the equality

$$
w_{A}(\lambda)=I_{m}-\Pi_{2}^{*} S^{-1}\left(A_{1}-\lambda I_{n}\right)^{-1} \Pi_{1},
$$

where the matrices $A_{1}, A_{2}, S, \Pi_{1}$, and $\Pi_{2}$ form an $S$-node, that is, satisfy the matrix (operator) identity

$$
A_{1} S-S A_{2}=\Pi_{1} \Pi_{2}^{*}
$$

Here $S$ and $A_{k}(k=1,2)$ are $n \times n$ matrices and $\Pi_{k}(k=1,2)$ are $n \times m$ matrices for some integer $n>0$. Sometimes we use notations $A_{1}=A$ and $\Pi_{1}=\Pi$. The term "transfer matrix function" can be explained via system theory [38] (see Introductions in [76, 77]). Namely, $w_{A}$ is the transfer matrix function of the system

$$
\frac{d}{d t} z=A_{1} z+\Pi_{1} \mu, \quad y=\Pi_{2}^{*} S^{-1} z+\mu,
$$

where $\mu(t) \in \mathbb{C}^{m}(t \geq 0)$ is the so called control. That is, for the Laplace transformations $y_{L}$ and $\mu_{L}$ of $y$ and $\mu$, respectively, we have $y_{L}(\lambda)=w_{A}(\lambda) \mu_{L}(\lambda)$. Indeed, if $z$ and $\mu$ satisfy some 
standard for Laplace transformations requirements and conditions

$$
\lim _{t \rightarrow \infty} e^{-\lambda t} z(t)=z(0)=0
$$

are fulfilled, we can apply Laplace transformations to both sides of equations in (1.7) and get

$$
\lambda z_{L}=A_{1} z_{L}+\Pi_{1} \mu_{L}, \quad y_{L}=\Pi_{2}^{*} S^{-1} z_{L}+\mu_{L}, \quad z_{L}:=\int_{0}^{\infty} e^{-\lambda t} z(t) d t .
$$

Now, the equality $y_{L}(\lambda)=w_{A}(\lambda) \mu_{L}(\lambda)$ is immediate from (1.5) and (1.8). As mentioned before, in this review we treat systems of the form $u_{x}=G(x, \lambda) u$ and system (1.7) is used only to explain the term "transfer matrix function". At the same time it is interesting that $w_{A}$ is a generalization of the Livšic-Brodskii characteristic matrix function.

Note that we consider transfer matrix functions $w_{A}(x, \lambda)$, which depend also on the variable $x$. More precisely $\Pi_{k}$ and $S$ in (1.5) depend on $x$. Matrix functions $\Pi_{k}(x)$ are introduced in terms of the generalized eigenfunction (with the generalized matrix eigenvalue) of the initial and dual to initial systems (see Remark 2.4 and formulas (3.3) and (3.4)). Recall that eigenfunctions are essential for the classical Bäcklund-Darboux transformation. Matrix function $S(x)$ is expressed directly via $\Pi_{k}(x)$, and in many cases one can use for this purpose the identity (1.6). Another type of matrix identities have been successfully used for the construction of the explicit solutions of nonlinear equations in [45]. Further developments of the Marchenko scheme are given in [11, 12, 78]. For application of the matrix identities to the construction of solitons see also [39].

Our approach grants additionally explicit expression for the Darboux matrix, allows to avoid the stages of the construction of the high order matrix solutions of the nonlinear equation and their reduction to the required order, and minimizes the order of the matrix that has to be inverted (matrix $S$ in our case).

GBDT for self-adjoint and skew-self-adjoint Dirac-type systems, for the system auxiliary to the $N$-wave equation, GBDT for the $N$-wave equation itself and for nonlinear Schrödinger equation are studied in the next Section "Preliminaries". A much more general Theorem 3.1 on GBDT and some applications are given in Section 3. Radial Dirac equation is treated in Section 4. Various direct and inverse problems are solved in Section 5.

By $\mathcal{I}$ and $\mathcal{I}_{k}$ we denote intervals on the real axis, by $\operatorname{diag}\left\{d_{1}, d_{2}, \ldots\right\}$ we denote diagonal matrix with the entries $d_{k}$ on the diagonal, by $[D, \xi]$ we denote the commutator $D \xi-\xi D$, and col means column. By the neighbourhood of zero we mean the neighbourhood of the form $(0, \varepsilon)$ or $[0, \varepsilon)$. As usual we denote by $\mathbb{Z}$ the integers, by $\mathbb{R}$ the real axis, by $\mathbb{C}$ the complex plain, by $\mathbb{R}_{+}$the positive semi-axis, and by $\mathbb{C}_{+}\left(\overline{\mathbb{C}}_{+}\right)$the open (closed) upper semi-plane. By $\arg (a)$ we denote the argument of $a \in \mathbb{C}$. We always assume that $\sum_{j=k}^{r} d_{j}=0$ when $k>r$. The notation $\tau \uparrow$ means that $\tau$ is a nondecreasing function. The spectrum of an operator $A$ is denoted by $\sigma(A)$. Matrices $j$ and $J$ have the form

$$
j=\left[\begin{array}{cc}
I_{p} & 0 \\
0 & -I_{p}
\end{array}\right], \quad J=\left[\begin{array}{cc}
0 & I_{p} \\
I_{p} & 0
\end{array}\right] .
$$




\section{Preliminaries}

In this section we apply GBDT to the self-adjoint and skew-self-adjoint Dirac-type systems and to the system auxiliary to the $N$-wave equation. We treat these systems on some interval $\mathcal{I}$ that contains 0 . Applications to the $N$-wave and nonlinear Schrödinger equations are obtained. In this way we can show how GBDT works before the formulation of more general results.

\subsection{Gauge transformation of the Dirac-type system}

A self-adjoint Dirac-type system has the form

$$
\frac{d}{d x} y(x, \lambda)=i(\lambda j+j V(x)) y(x, \lambda)
$$

where $y(x, \lambda) \in \mathbb{C}^{m}, m=2 p$,

$$
j=\left[\begin{array}{cc}
I_{p} & 0 \\
0 & -I_{p}
\end{array}\right], \quad V=\left[\begin{array}{cc}
0 & v \\
v^{*} & 0
\end{array}\right],
$$

and the potential $v$ is a $p \times p$ matrix function. A skew-self-adjoint Dirac-type system has the form

$$
\frac{d}{d x} y(x, \lambda)=(i \lambda j+j V(x)) y(x, \lambda) .
$$

Dirac-type systems are also called Dirac, Zakharov-Shabat or AKNS systems.

As mentioned already in the introduction, the $m \times m$ fundamental solutions of first order systems we usually denote by $u$ (or $\widetilde{u}$ ) and normalize by (1.4). Sometimes we write down the systems considered in this review in terms of $u$ or $\widetilde{u}$ at once. System (2.1) can be rewritten as:

$$
\frac{d}{d x} u(x, \lambda)+\left(\lambda q_{1}+q_{0}(x)\right) u(x, \lambda)=0, \quad q_{1} \equiv-i j, \quad q_{0}(x)=-i j V(x) .
$$

GBDT for system (2.4) is generated by an integer $n>0$ and three matrices, that is, by $n \times n$ matrices $A$ and $S(0)=S(0)^{*}$ and by $n \times m$ matrix $\Pi(0)$. It is required that these matrices satisfy the matrix identity

$$
A S(0)-S(0) A^{*}=i \Pi(0) j \Pi(0)^{*} .
$$

Fix $n$ and parameter matrices $A, S(0)$, and $\Pi(0)$. Then we define an $n \times m$ matrix function $\Pi(x)$ by its value $\Pi(0)$ at $x=0$ and by the linear differential equation

$$
\Pi_{x}(x)=A \Pi(x) q_{1}+\Pi(x) q_{0}(x) .
$$

Matrix function $S(x)$ is easily recovered from its value $S(0)$ and from the expression for its derivative:

$$
S_{x}=\Pi \Pi^{*} .
$$

Moreover, as $S(0)=S(0)^{*}$ and $S_{x}=S_{x}^{*}$ we have $S(x)=S(x)^{*}$. 
Recall that we consider systems and functions on the interval $\mathcal{I}$ :

$$
x \in \mathcal{I} \text { and } 0 \in \mathcal{I} \text {. }
$$

We choose point 0 , where $\Pi(x)$ and $S(x)$ are fixed, for convenience. (It could be any other point from $\mathcal{I}$.) In (2.7) and in some of the following formulas we omit the variables in the notations.

From (2.6) and (2.7) we get

$$
\begin{gathered}
\left(A S-S A^{*}\right)_{x}=A \Pi \Pi^{*}-\Pi^{*} A^{*}, \\
\left(i \Pi j \Pi^{*}\right)_{x}=\left(i \Pi_{x} j \Pi^{*}\right)-\left(i \Pi_{x} j \Pi^{*}\right)^{*}=A \Pi^{*}-\Pi^{*} A^{*}, \text { i.e. } \\
\left(A S-S A^{*}\right)_{x}=\left(i \Pi j \Pi^{*}\right)_{x} .
\end{gathered}
$$

Formulas (2.5) and (2.8) imply identity

$$
A S(x)-S(x) A^{*}=i \Pi(x) j \Pi(x)^{*} .
$$

That is, matrices $A, S(x)$, and $\Pi(x)$ satisfy identity (1.6), where

$$
A_{1}=A_{2}^{*}=A, \quad \Pi_{1}=\Pi, \quad \Pi_{2}^{*}=i j \Pi^{*},
$$

and we say that $A, S(x)$, and $\Pi(x)$ form an $S$-node. Using (2.10) rewrite formula (1.5) for the transfer matrix function

$$
w_{A}(x, \lambda)=I_{m}-i j \Pi(x)^{*} S(x)^{-1}\left(A-\lambda I_{n}\right)^{-1} \Pi(x) .
$$

Thus constructed matrix function $w_{A}$ is a gauge transformation of the Dirac type system. To show this we shall differentiate $w_{A}$ using (2.6), (2.7), and (2.9). First calculate the derivative of $\Pi^{*} S^{-1}$ :

$$
\left(\Pi^{*} S^{-1}\right)_{x}=i j \Pi^{*} A^{*} S^{-1}+i V j \Pi^{*} S^{-1}-\Pi^{*} S^{-1} \Pi \Pi^{*} S^{-1} .
$$

Taking into account that (2.9) yields $A^{*} S^{-1}=S^{-1} A-i S^{-1} \Pi \Pi^{*} S^{-1}$, we rewrite (2.12) as

$$
\left(\Pi^{*} S^{-1}\right)_{x}=i j \Pi^{*} S^{-1} A+\left(i V j+j \Pi^{*} S^{-1} \Pi j-\Pi^{*} S^{-1} \Pi\right) \Pi^{*} S^{-1} .
$$

Using (2.6), (2.11), and (2.13) one gets

$$
\begin{aligned}
\frac{d}{d x} w_{A}= & -i j\left(\left(i V j+j \Pi^{*} S^{-1} \Pi j-\Pi^{*} S^{-1} \Pi\right) \Pi^{*} S^{-1}\left(A-\lambda I_{n}\right)^{-1} \Pi\right. \\
& \left.+i j \Pi^{*} S^{-1}\left(A-\lambda I_{n}+\lambda I_{n}\right)\left(A-\lambda I_{n}\right)^{-1} \Pi\right) \\
& -i j \Pi^{*} S^{-1}\left(A-\lambda I_{n}\right)^{-1}\left(-i\left(A-\lambda I_{n}+\lambda I_{n}\right) \Pi j-i \Pi j V\right) .
\end{aligned}
$$

From (2.11) and (2.14) it follows that

$$
\begin{aligned}
\frac{d}{d x} w_{A}= & \left(i j V+\Pi^{*} S^{-1} \Pi-j \Pi^{*} S^{-1} \Pi j\right)\left(w_{A}-I_{n}\right)+\Pi^{*} S^{-1} \Pi \\
& +i \lambda j\left(w_{A}-I_{n}\right)-j \Pi^{*} S^{-1} \Pi j-\left(w_{A}-I_{n}\right)(i \lambda j+i j V) \\
= & \left(i \lambda j+i j V+\Pi^{*} S^{-1} \Pi-j \Pi^{*} S^{-1} \Pi j\right) w_{A}-w_{A}(i \lambda j+i j V) .
\end{aligned}
$$


Finally, rewrite relation above as

$$
\frac{d}{d x} w_{A}(x, \lambda)=i(\lambda j+j \widetilde{V}(x)) w_{A}(x, \lambda)-i w_{A}(x, \lambda)(\lambda j+j V(x)),
$$

where

$$
\begin{gathered}
\widetilde{V}=\left[\begin{array}{cc}
0 & \widetilde{v} \\
\widetilde{v}^{*} & 0
\end{array}\right]=V+i\left(\Pi^{*} S^{-1} \Pi j-j \Pi^{*} S^{-1} \Pi\right), \\
\widetilde{v}=v-2 i\left[\begin{array}{ll}
I_{p} & 0
\end{array}\right] \Pi^{*} S^{-1} \Pi\left[\begin{array}{c}
0 \\
I_{p}
\end{array}\right] .
\end{gathered}
$$

Thus the following proposition is proved.

Proposition 2.1. Let Dirac system (2.1) and parameter matrices $A, \Pi(0)$, and $S(0)=S(0)^{*}$ be given, and let equality (2.5) hold. Then the matrix function $w_{A}$ defined by (2.11), where $\Pi(x)$ and $S(x)$ are obtained via formulas (2.6) and (2.7), is a gauge transformation (Darboux matrix) of the Dirac system and satisfies equation (2.15). The fundamental solution of the transformed system $\frac{d}{d x} \widetilde{u}=i(\lambda j+j \widetilde{V}) \widetilde{u}$ is given by the formula

$$
\widetilde{u}(x, \lambda)=w_{A}(x, \lambda) u(x, \lambda) w_{A}(0, \lambda)^{-1},
$$

where $u$ is the fundamental solution of the initial system (2.1).

Recall that we normalize fundamental solutions $u$ by the condition (1.4).

The skew-self-adjoint Dirac-type system can be written as the first relation in (2.4), where $q_{1} \equiv-i j, q_{0}(x)=-j V(x)$, and we put

$$
A_{1}=A_{2}^{*}=A, \quad \Pi_{1}=\Pi, \quad \Pi_{2}^{*}=i \Pi^{*},
$$

where only the last equality differs from the last equality in (2.10). After substitution of our new $q_{0}$ we define $\Pi$ via (2.6):

$$
\Pi_{x}(x)=A \Pi(x) q_{1}+\Pi(x) q_{0}(x)=-i A \Pi(x) j-\Pi(x) j V(x) .
$$

We define $S$ by the equality

$$
S_{x}=\Pi_{1} q_{1} \Pi_{2}^{*}=\Pi j \Pi^{*} .
$$

Under these conditions and under the matrix identity condition at $x=0$

$$
A S(0)-S(0) A^{*}=i \Pi(0) \Pi(0)^{*},
$$

the identity

$$
A S(x)-S(x) A^{*}=i \Pi(x) \Pi(x)^{*}
$$

follows. Similar to the previous Proposition 2.1 our next proposition can be proved. 
Proposition 2.2. Let skew-self-adjoint Dirac-type system (2.3) be given, and let (2.22) hold. Then the matrix function

$$
w_{A}(x, \lambda)=I_{m}-i \Pi(x)^{*} S(x)^{-1}\left(A-\lambda I_{n}\right)^{-1} \Pi(x),
$$

where $\Pi$ and $S$ are defined by (2.20) and (2.21), is a gauge transformation of (2.3) and satisfies equation

$$
\frac{d}{d x} w_{A}(x, \lambda)=(i \lambda j+j \tilde{V}(x)) w_{A}(x, \lambda)-w_{A}(x, \lambda)(i \lambda j+j V(x)) .
$$

Here we have

$$
\widetilde{V}=\left[\begin{array}{cc}
0 & \widetilde{v} \\
\widetilde{v}^{*} & 0
\end{array}\right]=V+\Pi^{*} S^{-1} \Pi-j \Pi^{*} S^{-1} \Pi j .
$$

Proposition 2.2 admits generalization for the case of the system auxiliary to nonlinear optics equation

$$
\frac{d}{d x} y(x, \lambda)=(i \lambda D-[D, \xi(x)]) y(x, \lambda), \quad \xi^{*}=B \xi B,
$$

where $y(x, \lambda) \in \mathbb{C}^{m}$ and

$$
D=\operatorname{diag}\left\{d_{1}, d_{2}, \ldots, d_{m}\right\}=D^{*} ; B=\operatorname{diag}\left\{b_{1}, b_{2}, \ldots, b_{m}\right\}, b_{k}= \pm 1 .
$$

To present (2.27) as the first equality in (2.4) we should put $q_{1} \equiv-i D$ and $q_{0}(x)=[D, \xi(x)]$. Substitute these expressions for $q_{1}$ and $q_{0}$ into (2.6) to define $\Pi$, and define $S$ by the equality $S_{x}=\Pi D B \Pi^{*}$ for the derivative of $S$. The matrix identity $A S(x)-S(x) A^{*}=i \Pi(x) B \Pi(x)^{*}$ easily follows after we assume $A S(0)-S(0) A^{*}=i \Pi(0) B \Pi(0)^{*}$.

Proposition 2.3. Let system (2.27) be given. Then the matrix function

$$
w_{A}(x, \lambda)=I_{m}-i B \Pi(x)^{*} S(x)^{-1}\left(A-\lambda I_{n}\right)^{-1} \Pi(x)
$$

is a gauge transformation of (2.27) and satisfies equation

$$
\frac{d}{d x} w_{A}(x, \lambda)=(i \lambda D-[D, \widetilde{\xi}(x)]) w_{A}(x, \lambda)-w_{A}(x, \lambda)(i \lambda D-[D, \xi(x)]) .
$$

Here we have

$$
\widetilde{\xi}=\xi-B \Pi^{*} S^{-1} \Pi, \quad \widetilde{\xi^{*}}=B \widetilde{\xi} B .
$$

Propositions 2.1-2.3 are particular subcases of a general version of Bäcklund-Darboux transformation for systems depending rationally on the spectral parameter $\lambda$ (see Section 3.).

Remark 2.4. When $A$ is a scalar (i.e., $n=1$ ), then by (2.6) $A$ is an eigenvalue and $\Pi$ is an eigenfunction of the system $\widehat{y}_{x}=\left(\lambda q_{1}+q_{0}\right) \widehat{y}$, which is dual to system $y_{x}+\left(\lambda q_{1}+q_{0}\right) y=0$ that we consider in this subsection. In a more general situation $n \geq 1$, we call A a generalized matrix eigenvalue and we call $\Pi$ a generalized eigenfunction. 


\section{2. $\quad N$-wave equation and gauge transformation}

Consider $N$-wave (nonlinear optics) equation

$$
\left[D, \xi_{t}(x, t)\right]-\left[\widehat{D}, \xi_{x}(x, t)\right]=[[D, \xi(x, t)],[\widehat{D}, \xi(x, t)]] \quad\left(\xi_{t}=\frac{\partial}{\partial t} \xi\right)
$$

on the product $\mathcal{I}_{1} \times \mathcal{I}_{2}$ of intervals $\mathcal{I}_{1}$ and $\mathcal{I}_{2}$, where

$$
\xi^{*}=B \xi B, \quad(0,0) \in \mathcal{I}_{1} \times \mathcal{I}_{2}, \quad \widehat{D}=\operatorname{diag}\left\{\widehat{d}_{1}, \ldots, \widehat{d}_{m}\right\}=\widehat{D}^{*},
$$

and $D$ and $B$ are given by (2.28). Nonlinear integrable equation (2.32) is the compatibility condition of two auxiliary linear systems [21,83] (see also [2] for the case $N>3$ ):

$$
\begin{aligned}
& u_{x}(x, t, \lambda)=(i \lambda D-[D, \xi(x, t)]) u(x, t, \lambda), \\
& u_{t}(x, t, \lambda)=(i \lambda \widehat{D}-[\widehat{D}, \xi(x, t)]) u(x, t, \lambda) .
\end{aligned}
$$

Indeed, in view of (2.34) and (2.35) we have

$$
u_{x t}=-\left[D, \xi_{t}\right] u+(i \lambda D-[D, \xi])(i \lambda \widehat{D}-[\widehat{D}, \xi]) u
$$

and

$$
u_{t x}=-\left[\widehat{D}, \xi_{x}\right] u+(i \lambda \widehat{D}-[\widehat{D}, \xi])(i \lambda D-[D, \xi]) u .
$$

Thus one can easily see that the compatibility condition $u_{x t}=u_{t x}$ is equivalent to (2.32).

To construct gauge transformation we fix $n>0$ and three parameter matrices, that is, two $n \times n$ matrices $A$ and $S(0,0)=S(0,0)^{*}$, and an $n \times m$ matrix $\Pi(0,0)$ such that

$$
A S(0,0)-S(0,0) A^{*}=i \Pi(0,0) B \Pi(0,0)^{*} .
$$

Now, introduce matrix functions $\Pi(x, t)$ and $S(x, t)$ by the equations

$$
\begin{array}{cl}
\Pi_{x}=-i A \Pi D+\Pi[D, \xi], & \Pi_{t}=-i A \Pi \widehat{D}+\Pi[\widehat{D}, \xi], \\
S_{x}=\Pi D B \Pi^{*}, \quad S_{t}=\Pi \widehat{D} B \Pi^{*} .
\end{array}
$$

Quite similar to $u_{x t}=u_{t x}$ one can show that according to (2.32) equations (2.37) are compatible, i.e., $\Pi_{x t}=\Pi_{t x}$. By (2.37) and (2.38) it is immediate that $S_{x t}=S_{t x}$. Proposition 2.3 implies

Proposition 2.5. Let $m \times m$ continuously differentiable matrix function $\xi\left(\xi^{*}=B \xi B\right)$ satisfy $N$-wave equation (2.32) and let matrix functions $\Pi$ and $S=S^{*}$ satisfy (2.36)-(2.38). Then in the points of invertibility of $S$ the matrix function

$$
\widetilde{\xi}(x, t):=\xi(x, t)-B \Pi(x, t)^{*} S(x, t)^{-1} \Pi(x, t) .
$$

satisfies equation (2.32) and an additional condition $\widetilde{\xi}^{*}=B \widetilde{\xi} B$. 
Proof. Equality $\widetilde{\xi} *=B \widetilde{\xi} B$ is immediate. Now, let $u$ be the fundamental solution of (2.34) and (2.35), i.e., let $u$ satisfy equations (2.34) and (2.35), and equality $u(0,0, \lambda)=I_{m}$. (As $\xi$ satisfies (2.32), the compatibility condition for systems (2.34) and (2.35) is fulfilled, and such a matrix function $u$ exists. See, for instance, formula (1.6) on p.168 in [77].) Put

$$
w_{A}(x, t, \lambda)=I_{m}-i B \Pi(x, t)^{*} S(x, t)^{-1}\left(A-\lambda I_{n}\right)^{-1} \Pi(x, t),
$$

and calculate derivatives of $w_{A}$ with respect to $x$ and $t$ using Proposition 2.3 in both cases. Then, for $\widetilde{u}(x, t, \lambda)=w_{A}(x, t, \lambda) u(x, t, \lambda)$ we have

$$
\widetilde{u}_{x}(x, t, \lambda)=\widetilde{G}(x, t, \lambda) \widetilde{u}(x, t, \lambda), \quad \widetilde{u}_{t}(x, t, \lambda)=\widetilde{F}(x, t, \lambda) \widetilde{u}(x, t, \lambda),
$$

where

$$
\widetilde{G}=i \lambda D-[D, \widetilde{\xi}], \quad \widetilde{F}=i \lambda \widehat{D}-[\widehat{D}, \widetilde{\xi}] .
$$

From the continuous differentiability of $\xi$ follows the continuous differentiability of $\widetilde{\xi}$ in the points of invertibility of $S$. Hence, in view of (2.41) we get $\widetilde{u}_{x t}=\widetilde{u}_{t x}$ or equivalently $\widetilde{G}_{t}-\widetilde{F}_{x}+[\widetilde{G}, \widetilde{F}]=0$ (see also the proof of Theorem 3.7). As we have already discussed here, the last equality is in its turn equivalent to the $N$-wave equation $\left[D, \widetilde{\xi}_{t}\right]-\left[\widehat{D}, \widetilde{\xi}_{x}\right]=[[D, \widetilde{\xi}],[\widehat{D}, \widetilde{\xi}]]$.

In the case of the trivial initial solution $\xi \equiv 0$ we obtain explicit solutions of the $N$-wave equation. Namely, putting $\Pi(0,0)=\left[\begin{array}{llll}f_{1} & f_{2} & \ldots & f_{m}\end{array}\right]$ and using (2.37) we recover $\Pi$ :

$$
\Pi(x, t)=\left[\exp \left(-i\left(d_{1} x+\widehat{d}_{1} t\right) A\right) f_{1} \quad \exp \left(-i\left(d_{2} x+\widehat{d}_{2} t\right) A\right) f_{2} \quad \ldots\right] .
$$

Next we recover $S$, and explicit formulas for solutions of the $N$-wave equation are immediate from (2.39).

\subsection{Nonlinear Schrödinger equation: n-modulation solutions}

Auxiliary systems (2.34) and (2.35) are a particular case of the linear differential first order systems

$$
u_{x}(x, t, \lambda)=G(x, t, \lambda) u(x, t, \lambda), \quad u_{t}(x, t, \lambda)=F(x, t, \lambda) u(x, t, \lambda) .
$$

The compatibility condition for systems (2.44) is given by the equation

$$
G_{t}-F_{x}+[G, F]=0 .
$$

When the first and second system in (2.44) takes the form (2.34) and (2.35), correspondingly, the compatibility condition (2.45) is equivalent to $N$-wave equation (2.32).

In this subsection we consider another example, namely, the well-known integrable [86] focusing nonlinear Schrödinger equation (fNLS)

$$
2 v_{t}+i\left(v_{x x}+2 v v^{*} v\right)=0 .
$$


Here $v(x, t)$ is a $p \times p$ matrix function. Equation (2.46) is the compatibility condition of the auxiliary systems (2.44), where

$$
G=i \lambda j+j V, \quad F=i\left(\lambda^{2} j-i \lambda j V-\left(V_{x}+j V^{2}\right) / 2\right),
$$

and $j$ and $V(x, t)$ are defined in (2.2). Consider again domain $\mathcal{I}_{1} \times \mathcal{I}_{2}$, where $(0,0) \in \mathcal{I}_{1} \times \mathcal{I}_{2}$. Our next proposition is a particular case of the results of Section 3. that can be proved similar to Propositions 2.1, 2.5.

Proposition 2.6. Let $p \times p$ matrix function $v(x, t)$ satisfy equation (2.46) and be continuously differentiable together with $v_{x}$. Let $n \times m$ matrix function $\Pi$ and $n \times n$ matrix function $S=S^{*}$ satisfy equations

$$
\begin{gathered}
A S(0,0)-S(0,0) A^{*}=i \Pi(0,0) \Pi(0,0)^{*}, \\
\Pi_{x}=-i A \Pi j-\Pi j V, \quad \Pi_{t}=-i A^{2} \Pi j-A \Pi j V+\frac{i}{2} \Pi\left(V_{x}+j V^{2}\right), \\
S_{x}=\Pi j \Pi^{*}, \quad S_{t}=A \Pi j \Pi^{*}+\Pi j \Pi^{*} A^{*}-i \Pi j V \Pi^{*},
\end{gathered}
$$

where $A$ is an $n \times n$ parameter matrix. Then the identity

$$
A S(x, t)-S(x, t) A^{*}=i \Pi(x, t) \Pi(x, t)^{*}
$$

holds in the domain $\mathcal{I}_{1} \times \mathcal{I}_{2}$, and the matrix function

$$
\widetilde{v}=v+2\left[\begin{array}{ll}
I_{p} & 0
\end{array}\right] \Pi^{*} S^{-1} \Pi\left[\begin{array}{c}
0 \\
I_{p}
\end{array}\right]
$$

satisfies (2.46) in the points of invertibility of $S$. Moreover, the transfer matrix function $w_{A}$ given by (2.24) satisfies equations $\left(w_{A}\right)_{x}=\widetilde{G} w_{A}-w_{A} G,\left(w_{A}\right)_{t}=\widetilde{F} w_{A}-w_{A} F$, where $\widetilde{G}$ and $\widetilde{F}$ are obtained by substitution of $\widetilde{V}=\left[\begin{array}{cc}0 & \widetilde{v} \\ \widetilde{v}^{*} & 0\end{array}\right]$ instead of $V$ into the right-hand sides of the first and second, respectively, relations in (2.47).

In the previous subsection we constructed explicit solutions of the $N$-wave equation, using trivial initial solution $\xi=0$. In the same way the $N$-soliton solutions of the fNLS can also be constructed [29, 55], putting $v=0$ in (2.49), (2.50), and (2.52). Now, we shall study a slightly more complicated situation, that is, the case of the nontrivial background .

Example 2.7. Let $p=1$, and $v=e^{-i t}$. One easily checks that

$$
u(x, t, \lambda)=(\exp (-i t j / 2)) C_{0}(\lambda) \exp \left((x+\lambda t) C_{1}(\lambda)\right),
$$

where

$$
C_{0}(\lambda)=\left[\begin{array}{lr}
1 & 1 \\
-i\left(\sqrt{1+\lambda^{2}}+\lambda\right) & i\left(\sqrt{1+\lambda^{2}}-\lambda\right)
\end{array}\right], \quad C_{1}(\lambda)=-i\left(\sqrt{1+\lambda^{2}}\right) j
$$


satisfies (2.44), (2.47) for the case $v=e^{-i t}$. Here, $u$ is not normalized (i.e., $\left.u(0,0, \lambda) \neq I_{2}\right)$. Choose for simplicity

$$
A=\operatorname{diag}\left\{a_{1}, a_{2}, \ldots, a_{n}\right\}, \quad a_{k} \neq \overline{a_{l}} \quad(k, l \leq n),
$$

and put

$$
\psi_{k}(x, t)=\left(u\left(x, t, \overline{a_{k}}\right) f_{k}\right)^{*}, \quad f_{k} \in \mathbb{C}^{2}, \quad f_{k} \neq 0 .
$$

It follows from (2.44), (2.47), and (2.56) that

$$
\left(\psi_{k}^{*}\right)_{x}=i \overline{a_{k}} j \psi_{k}^{*}+j V \psi_{k}^{*}, \quad\left(\psi_{k}^{*}\right)_{t}=i{\overline{a_{k}}}^{2} j \psi_{k}^{*}+\overline{a_{k}} j V \psi_{k}^{*}-\frac{i}{2} j V^{2} \psi_{k}^{*} .
$$

Taking into account that $v_{x}=0$ (i.e., $V_{x}=0$ ), by (2.55) and (2.57) we see that the matrix function

$$
\Pi=\left[\begin{array}{c}
\psi_{1} \\
\cdots \\
\psi_{n}
\end{array}\right]
$$

satisfies (2.49). Thus $\Pi$ is obtained from (2.56) and (2.58), and then relations

$$
S=\left\{s_{k j}\right\}_{k, j=1}^{n}, \quad s_{k j}=i \psi_{k} \psi_{j}^{*} /\left(a_{k}-\overline{a_{j}}\right)
$$

follow from the matrix identity (2.51). In this way, using (2.52) matrix function $\widetilde{v}$ is constructed explicitly.

Fix now integers $\left\{r_{1 k}\right\},\left\{r_{2 k}\right\}$ such that $r_{1 k}^{2}-r_{2 k}^{2}=l_{k}^{2}(1 \leq k \leq n)$, where $l_{k}$ are integer, and put $a_{k}=i r_{1 k} / r_{2 k}$. Then, by (2.53) and (2.56) the dependence of $\psi_{k}^{*}$ on $t$ can be expressed in terms of functions $\exp ( \pm i t / 2)$ and $\exp \left( \pm i l_{k} r_{1 k} t / r_{2 k}^{2}\right)$. Therefore, taking into account (2.52), (2.58), and (2.59) we see that $\widetilde{v}$ is a periodical in $t$ solution.

Under somewhat more restrictive than (2.55) conditions

$$
A=\operatorname{diag}\left\{a_{1}, a_{2}, \ldots, a_{n}\right\}, \quad \sigma(A) \in \mathbb{C}_{+}, \quad a_{k} \neq a_{l}(k \neq l),
$$

the matrix function $S(x, t)$ is always invertible, and moreover we have $S(x, t)>0$. To show this we rewrite identity (2.51) in the form

$$
S\left(A^{*}-\lambda I_{n}\right)^{-1}-\left(A-\lambda I_{n}\right)^{-1} S=i\left(A-\lambda I_{n}\right)^{-1} \Pi \Pi^{*}\left(A^{*}-\lambda I_{n}\right)^{-1} .
$$

As $\sigma(A) \in \mathbb{C}_{+}$, by the theorem on residues one represents $S$ as integrals of the right-hand side of (2.61) on contours in $\overline{\mathbb{C}}_{+}$, and in the limit we get

$$
S=\frac{1}{2 \pi} \int_{-\infty}^{\infty}\left(A-\lambda I_{n}\right)^{-1} \Pi \Pi^{*}\left(A^{*}-\lambda I_{n}\right)^{-1} d \lambda,
$$

that is, $S \geq 0$. To derive the strict inequality suppose $S g=0, g=\left\{g_{j}\right\}_{j=1}^{n} \neq 0$. Hence we have $g^{*}\left(A S-S A^{*}\right) g=0$, and so by (2.51) the equality $g^{*} \Pi \Pi^{*} g=0$ holds, i.e., $\Pi^{*} g=0$. Using again (2.51) one gets $S A^{*} g=0$. Now, by induction equalities $S\left(A^{*}\right)^{k} g=0, \Pi^{*}\left(A^{*}\right)^{k} g=0(k \geq 0)$ easily follow. As by our assumption $g \neq 0$, there is its entry $g_{r} \neq 0$. In view of the third relation in (2.60) we obtain $e(r):=\left\{\delta_{r j}\right\}_{j=1}^{n} \in$ span $\bigcup_{k=0}^{n-1}\left(A^{*}\right)^{k} g$, where $\delta_{r j}$ is the Kronecker-symbol. Therefore from $\Pi^{*}\left(A^{*}\right)^{k} g=0(k \geq 0)$ it follows $\Pi^{*} e(r)=0$, that is, $\psi_{r}=0$. The last equality contradicts (2.56), and so inequality $S>0$ is proved. Taking into account $S>0$ we see that the fNLS solutions given by (2.52) are well-defined. 


\section{GBDT for system depending rationally on spectral parameter and explicit solutions of nonlinear equations}

\subsection{GBDT for system depending rationally on $\lambda$}

In this section we consider GBDT for a general case of first order system depending rationally on the spectral parameter $\lambda$ :

$$
u_{x}=G u, \quad G(x, \lambda)=-\left(\sum_{k=0}^{r} \lambda^{k} q_{k}(x)+\sum_{s=1}^{l} \sum_{k=1}^{r_{s}}\left(\lambda-c_{s}\right)^{-k} q_{s k}(x)\right),
$$

$x \in \mathcal{I}$, where $\mathcal{I}$ is an interval such that $0 \in \mathcal{I}$, and the coefficients $q_{k}(x)$ and $q_{s k}(x)$ are $m \times m$ locally integrable matrix functions.

As before we fix an integer $n>0$. Next, we fix five matrices, namely, $n \times n$ matrices $A_{k}$ $(k=1,2)$ and $S(0)$, and $n \times m$ matrices $\Pi_{k}(0)(k=1,2)$. It is required that these matrices form an $S$-node, that is, the identity

$$
A_{1} S(0)-S(0) A_{2}=\Pi_{1}(0) \Pi_{2}(0)^{*}
$$

holds. Matrix functions $\Pi_{k}(x)$ are introduced via the coefficients from $G$ :

$$
\begin{aligned}
& \left(\Pi_{1}\right)_{x}=\sum_{k=0}^{r} A_{1}^{k} \Pi_{1} q_{k}+\sum_{s=1}^{l} \sum_{k=1}^{r_{s}}\left(A_{1}-c_{s} I_{n}\right)^{-k} \Pi_{1} q_{s k}, \\
& \left(\Pi_{2}^{*}\right)_{x}=-\left(\sum_{k=0}^{r} q_{k} \Pi_{2}^{*} A_{2}^{k}+\sum_{s=1}^{l} \sum_{k=1}^{r_{s}} q_{s k} \Pi_{2}^{*}\left(A_{2}-c_{s} I_{n}\right)^{-k}\right) .
\end{aligned}
$$

Compare (3.1) with (3.4) to see that $\Pi_{2}^{*}$ can be viewed as a generalized eigenfunction of the system $u_{x}=G u$.

Matrix function $S(x)$ is introduced via $\frac{d}{d x} S$ by the equality

$$
\begin{aligned}
S_{x}= & \sum_{k=1}^{r} \sum_{j=1}^{k} A_{1}^{k-j} \Pi_{1} q_{k} \Pi_{2}^{*} A_{2}^{j-1}-\sum_{s=1}^{l} \sum_{k=1}^{r_{s}} \sum_{j=1}^{k}\left(A_{1}-c_{s} I_{n}\right)^{j-k-1} \\
& \times \Pi_{1} q_{s k} \Pi_{2}^{*}\left(A_{2}-c_{s} I_{n}\right)^{-j} .
\end{aligned}
$$

Equality (3.5) is chosen so that the identity $\left(A_{1} S-S A_{2}\right)_{x}=\left(\Pi_{1} \Pi_{2}^{*}\right)_{x}$ holds. Hence, taking into account (3.2) we have

$$
A_{1} S(x)-S(x) A_{2}=\Pi_{1}(x) \Pi_{2}(x)^{*}, \quad x \in \mathcal{I} .
$$

By Theorem 3.1 below, the Darboux matrix for system (3.1) has the form (1.5) :

$$
w_{A}(x, \lambda)=I_{m}-\Pi_{2}(x)^{*} S(x)^{-1}\left(A_{1}-\lambda I_{n}\right)^{-1} \Pi_{1}(x) .
$$


In other words, $w_{A}$ satisfies the equation

$$
\frac{d}{d x} w_{A}(x, \lambda)=\widetilde{G}(x, \lambda) w_{A}(x, \lambda)-w_{A}(x, \lambda) G(x, \lambda)
$$

where $\widetilde{G}$ has the same structure as $G$ :

$$
\widetilde{G}(x, \lambda)=-\left(\sum_{k=0}^{r} \lambda^{k} \widetilde{q}_{k}(x)+\sum_{s=1}^{l} \sum_{k=1}^{r_{s}}\left(\lambda-c_{s}\right)^{-k} \widetilde{q}_{s k}(x)\right) .
$$

The transformed coefficients $\widetilde{q}_{k}$ and $\widetilde{q}_{s k}$ are given by the formulas

$$
\begin{gathered}
\widetilde{q}_{k}=q_{k}-\sum_{j=k+1}^{r}\left(q_{j} Y_{j-k-1}-X_{j-k-1} q_{j}+\sum_{i=k+2}^{j} X_{j-i} q_{j} Y_{i-k-2}\right), \\
\widetilde{q}_{s k}=q_{s k}+\sum_{j=k}^{r_{s}}\left(q_{s j} Y_{s, k-j-1}-X_{s, k-j-1} q_{s j}-\sum_{i=k}^{j} X_{s, i-j-1} q_{s j} Y_{s, k-i-1}\right),
\end{gathered}
$$

where $X_{k}(x), Y_{k}(x), X_{s k}(x)$, and $Y_{s k}(x)$ are expressed in terms of the matrices $A_{k}$ and matrix functions $S(x)$ and $\Pi_{k}(x)$ :

$$
\begin{aligned}
& X_{k}=\Pi_{2}^{*} S^{-1} A_{1}^{k} \Pi_{1}, \quad Y_{k}=\Pi_{2}^{*} A_{2}^{k} S^{-1} \Pi_{1}, \\
& X_{s k}=\Pi_{2}^{*} S^{-1}\left(A_{1}-c_{s} I_{n}\right)^{k} \Pi_{1}, \quad Y_{s k}=\Pi_{2}^{*}\left(A_{2}-c_{s} I_{n}\right)^{k} S^{-1} \Pi_{1} .
\end{aligned}
$$

Theorem 3.1. [57] Let first order system (3.1) and five matrices $S(0), A_{k}$, and $\Pi_{k}(k=1,2)$ be given. Assume that the identity (3.2) holds and that $\left\{c_{s}\right\} \cap \sigma\left(A_{k}\right)=\emptyset(k=1,2)$. Then, in the points of invertibility of $S$, the transfer matrix function $w_{A}$ given by (3.7), where $S$ and $\Pi_{k}$ are determined by (3.3)-(3.5), satisfies equation (3.8), where $\widetilde{G}$ is determined by the formulas (3.9)-(3.13).

The proof of Theorem 3.1 for the case of one pole, that is, for $G(x, \lambda)=\sum_{k=-r}^{r} \lambda^{k} q_{k}$ is contained in [61]. The case of several poles $c_{s}$ can be treated precisely in the same way. The following formula is essential for the proof and is also of independent interest:

$$
\left(\Pi_{2}^{*} S^{-1}\right)_{x}=-\left(\sum_{k=0}^{r} \widetilde{q}_{k} \Pi_{2}^{*} S^{-1} A_{1}^{k}+\sum_{s=1}^{l} \sum_{k=1}^{r_{s}} \widetilde{q}_{s k} \Pi_{2}^{*} S^{-1}\left(A_{1}-c_{s} I_{n}\right)^{-k}\right) .
$$

Formula (3.14) means that multiplying $\Pi_{2}^{*}$ by $S^{-1}$ from the right we transform a generalized eigenfunction of system (3.1) into a generalized eigenfunction of the transformed system $\widetilde{u}_{x}=\widetilde{G} \widetilde{u}$. (Compare formula (3.14) with formula (3.4).)

Remark 3.2. It is immediate from (3.10) and (3.12), respectively, that $\widetilde{q}_{r}=q_{r}$ and $X_{0}=Y_{0}$.

Remark 3.3. If $\sigma\left(A_{1}\right) \cap \sigma\left(A_{2}\right)=\emptyset$ the matrix function $S(x)$ is uniquely defined by the matrix identity (3.6). 
Remark 3.4. In the points of the invertibility of $S$ and for $\lambda \notin\left(\sigma\left(A_{1}\right) \cup \sigma\left(A_{2}\right)\right)$ the matrix function $w_{A}(x, \lambda)$ is well-defined and invertible. Indeed, from the realization (3.7) and formula (A4) it follows that

$$
w_{A}(x, \lambda)^{-1}=I_{m}+\Pi_{2}(x)^{*} S(x)^{-1}\left(A^{\times}-\lambda I_{n}\right)^{-1} \Pi_{1}(x),
$$

where $A^{\times}=A_{1}-\Pi_{1} \Pi_{2}^{*} S^{-1}$. In view of (3.6) it is immediate that $A^{\times}=S A_{2} S^{-1}$. Hence, we get

$$
w_{A}(x, \lambda)^{-1}=I_{m}+\Pi_{2}(x)^{*}\left(A_{2}-\lambda I_{n}\right)^{-1} S(x)^{-1} \Pi_{1}(x) .
$$

\subsection{Explicit solutions of nonlinear equations}

One can apply Theorem 3.1 to construct solutions of nonlinear integrable equations and corresponding wave functions similar to the way, in which it was done in subsections 2.2. and 2.3. For this purpose we use auxiliary linear systems for integrable nonlinear equation:

$$
\begin{aligned}
& u_{x}=G u, \quad u_{t}=F u ; \\
& G(x, t, \lambda)=-\sum_{k=0}^{r} \lambda^{k} q_{k}(x, t)-\sum_{s=1}^{l} \sum_{k=1}^{r_{s}}\left(\lambda-c_{s}\right)^{-k} q_{s k}(x, t), \\
& F(x, t, \lambda)=-\sum_{k=0}^{R} \lambda^{k} Q_{k}(x, t)-\sum_{s=1}^{L} \sum_{k=1}^{R_{s}}\left(\lambda-C_{s}\right)^{-k} Q_{s k}(x, t),
\end{aligned}
$$

and zero curvature (compatibility condition) representation (2.45) of the integrable nonlinear equation itself. We consider nonlinear equations in the domain $(x, t) \in \mathcal{I}_{1} \times \mathcal{I}_{2}$ and assume $(0,0) \in$ $\mathcal{I}_{1} \times \mathcal{I}_{2}$.

Remark 3.5. If $G$ and $F$ are continuously differentiable and (2.45) holds, then according to formula (1.6) on p.168 in [77] there is the $m \times m$ solution $u$ of (3.16) normalized by the condition $u(0,0, \lambda)=I_{m}$.

Remark 3.6. Usually we shall asume that $G$ and $F$ are continuously differentiable and that $u(x, t, \lambda)$ is the solution of (3.16) normalized as in Remark 3.5.

When we deal with two auxiliary linear systems, the $n \times n$ matrix functions $S$ and the $n \times m$ matrix functions $\Pi_{k}$ depend on two variables $x$ and $t$, and the matrix identity (3.2) for parameter matrices $A_{k}, \Pi_{k}(0)$, and $S(0)$ is substituted by the identity

$$
A_{1} S(0,0)-S(0,0) A_{2}=\Pi_{1}(0,0) \Pi_{2}(0,0)^{*}
$$

for parameter matrices $A_{k}, \Pi_{k}(0,0)$, and $S(0,0)$. Equations (3.3)-(3.5) should be completed by the similar equations with respect to derivatives in $t$ :

$$
\begin{aligned}
& \left(\Pi_{1}\right)_{t}=\sum_{k=0}^{R} A_{1}^{k} \Pi_{1} Q_{k}+\sum_{s=1}^{L} \sum_{k=1}^{R_{s}}\left(A_{1}-C_{s} I_{n}\right)^{-k} \Pi_{1} Q_{s k}, \\
& \left(\Pi_{2}^{*}\right)_{t}=-\left(\sum_{k=0}^{R} Q_{k} \Pi_{2}^{*} A_{2}^{k}+\sum_{s=1}^{L} \sum_{k=1}^{R_{s}} Q_{s k} \Pi_{2}^{*}\left(A_{2}-C_{s} I_{n}\right)^{-k}\right),
\end{aligned}
$$




$$
\begin{aligned}
S_{t}= & \sum_{k=1}^{R} \sum_{j=1}^{k} A_{1}^{k-j} \Pi_{1} Q_{k} \Pi_{2}^{*} A_{2}^{j-1}-\sum_{s=1}^{L} \sum_{k=1}^{R_{s}} \sum_{j=1}^{k}\left(A_{1}-C_{s} I_{n}\right)^{j-k-1} \\
& \times \Pi_{1} Q_{s k} \Pi_{2}^{*}\left(A_{2}-C_{s} I_{n}\right)^{-j} .
\end{aligned}
$$

We require

$$
\left\{c_{s}\right\} \cap \sigma\left(A_{k}\right)=\emptyset, \quad\left\{C_{s}\right\} \cap \sigma\left(A_{k}\right)=\emptyset \quad(k=1,2) .
$$

Then Theorem 3.1 provides expessions for derivatives $\left(w_{A}(x, t, \lambda)\right)_{x}$ and $\left(w_{A}(x, t, \lambda)\right)_{t}$, where

$$
w_{A}(x, t, \lambda)=I_{m}-\Pi_{2}(x, t)^{*} S(x, t)^{-1}\left(A_{1}-\lambda I_{n}\right)^{-1} \Pi_{1}(x, t) .
$$

Hence, equations

$$
\widetilde{u}_{x}=\widetilde{G} \widetilde{u}, \quad \widetilde{u}_{t}=\widetilde{F} \widetilde{u}, \quad \widetilde{u}(x, t, \lambda):=w_{A}(x, t, \lambda) u(x, t, \lambda)
$$

hold. Finally in a way which is similar to the proof of (3.6), one can show that

$$
A_{1} S(x, t)-S(x, t) A_{2}=\Pi_{1}(x, t) \Pi_{2}(x, t)^{*}, \quad(x, t) \in \mathcal{I}_{1} \times \mathcal{I}_{2} .
$$

It follows from (3.25) that

$$
\widetilde{u}_{x t}=\left(\widetilde{G}_{t}+\widetilde{G} \widetilde{F}\right) \widetilde{u}, \quad \widetilde{u}_{t x}=\left(\widetilde{F}_{x}+\widetilde{F} \widetilde{G}\right) \widetilde{u} .
$$

If $G$ and $F$ are continuously differentiable, then $\widetilde{G}$ and $\widetilde{F}$ are continuously differentiable too. Hence, $\widetilde{u}_{t x}=\widetilde{u}_{x t}$ and formula (3.27) implies

$$
\left(\widetilde{G}_{t}-\widetilde{F}_{x}+[\widetilde{G}, \widetilde{F}]\right) \widetilde{u}=0 .
$$

By Remark 3.4 and identity (3.26) the matrix function $w_{A}(x, t, \lambda)$ is invertible, and by Remark 3.5 $u$ is invertible. Thus, $\widetilde{u}$ is invertible. Therefore, it is immediate from (3.28) that

$$
\widetilde{G}_{t}-\widetilde{F}_{x}+[\widetilde{G}, \widetilde{F}]=0
$$

For the particular case of the $N$-wave equation formula (3.29) was obtained in the proof of Proposition 2.5 and was used there to show that $\widetilde{\xi}$ satisfies the $N$-wave equation. Now, we proved the following general theorem.

Theorem 3.7. Let $G$ and $F$ be continuously differentiable and satisfy (2.45). Let the identity (3.19) and relation (3.23) hold, and let the matrix functions $\Pi_{k}$ and $S$ be given by the equations (3.3)(3.5) and (3.20)-(3.22). Then in the points of the invertibility of $S$ the zero curvature equation (3.29), where $\widetilde{G}$ and $\widetilde{F}$ are given by (3.9)-(3.13), holds.

Example 3.8. The main chiral field equation for $m \times m$ invertible matrix function $z$ has the form

$$
2 z_{x t}(x, t)=z_{x}(x, t) z(x, t)^{-1} z_{t}(x, t)+z_{t}(x, t) z(x, t)^{-1} z_{x}(x, t),
$$


and is equivalent [50, 84] to the compatibility condition (2.45) of the auxiliary systems (3.16), where

$$
\begin{aligned}
& G(x, t, \lambda)=-(\lambda-1)^{-1} q_{11}(x, t), \quad q_{11}=z_{x} z^{-1} \\
& F(x, t, \lambda)=-(\lambda+1)^{-1} Q_{11}(x, t), \quad Q_{11}=-z_{t} z^{-1} .
\end{aligned}
$$

Let z satisfy (3.30). In view of (3.31) and (3.32) equations (3.3)-(3.5) take the form

$$
\begin{gathered}
\left(\Pi_{1}\right)_{x}=\left(A_{1}-I_{n}\right)^{-1} \Pi_{1} z_{x} z^{-1}, \quad\left(\Pi_{2}^{*}\right)_{x}=-z_{x} z^{-1} \Pi_{2}^{*}\left(A_{2}-I_{n}\right)^{-1}, \\
S_{x}=-\left(A_{1}-I_{n}\right)^{-1} \Pi_{1} z_{x} z^{-1} \Pi_{2}^{*}\left(A_{2}-I_{n}\right)^{-1},
\end{gathered}
$$

and equations (3.20)-(3.22) take the form

$$
\begin{gathered}
\left(\Pi_{1}\right)_{t}=-\left(A_{1}+I_{n}\right)^{-1} \Pi_{1} z_{t} z^{-1}, \quad\left(\Pi_{2}^{*}\right)_{x}=z_{t} z^{-1} \Pi_{2}^{*}\left(A_{2}+I_{n}\right)^{-1}, \\
S_{t}=\left(A_{1}+I_{n}\right)^{-1} \Pi_{1} z_{t} z^{-1} \Pi_{2}^{*}\left(A_{2}+I_{n}\right)^{-1} .
\end{gathered}
$$

Now, let matrices $A_{k}, S(0,0)$, and $\Pi_{k}(0,0)$ be fixed. Assume that (3.19) holds and that $\pm 1 \notin \sigma\left(A_{k}\right)$ $(k=1,2)$. Let matrix functions $S$ and $\Pi_{k}$ satisfy (3.33)-(3.36). Taking into account (3.9) and first equalities in (3.31) and (3.32) we have

$$
\widetilde{G}(x, t, \lambda)=-(\lambda-1)^{-1} \widetilde{q}_{11}(x, t), \quad \widetilde{F}(x, t, \lambda)=-(\lambda+1)^{-1} \widetilde{Q}_{11}(x, t) .
$$

By Theorem 3.1 we get

$$
\left(w_{A}\right)_{x}=\widetilde{G} w_{A}-w_{A} G, \quad\left(w_{A}\right)_{t}=\widetilde{F} w_{A}-w_{A} F .
$$

Assume additionally that $\operatorname{det} A_{k} \neq 0(k=1,2)$. Then, by (3.15) and (3.24) the matrix functions $w_{A}(x, t, 0)$ and $w_{A}(x, t, 0)^{-1}$ are well-defined in the points of invertibility of $S$.

It follows from (3.37) and (3.38) that

$$
\begin{aligned}
\frac{\partial}{\partial x} w_{A}(x, t, 0) & =\widetilde{q}_{11}(x, t) w_{A}(x, t, 0)-w_{A}(x, t, 0) q_{11}(x, t), \\
\frac{\partial}{\partial t} w_{A}(x, t, 0) & =-\widetilde{Q}_{11}(x, t) w_{A}(x, t, 0)+w_{A}(x, t, 0) Q_{11}(x, t) .
\end{aligned}
$$

Rewrite second relations in (3.31) and (3.32):

$$
z_{x}=q_{11} z, \quad z_{t}=-Q_{11} z
$$

Put

$$
\widetilde{z}(x, t):=w_{A}(x, t, 0) z(x, t) .
$$

From formulas (3.39)-(3.42) we derive $\widetilde{z}_{x}=\widetilde{q}_{11} \widetilde{z}$ and $\widetilde{z}_{t}=-\widetilde{Q}_{11} \widetilde{z}$. As $w_{A}(x, t, 0)$ and $z(x, t)$ are invertible, so $\widetilde{z}$ is invertible, and we get

$$
\widetilde{q}_{11}=\widetilde{z}_{x} \widetilde{z}^{-1}, \quad \widetilde{Q}_{11}=-\widetilde{z}_{t} \widetilde{z}^{-1} .
$$


Recall that if formulas (3.31) and (3.32) hold, then (2.45) is equivalent to (3.30). The only difference between equalities in (3.31), (3.32) and equalities in (3.37), (3.43) is "tilde" in the notations. Hence, in view of (3.37) and (3.43) formula (3.29) implies that $\widetilde{z}$ satisfies main chiral field equation, that is,

$$
2 \widetilde{z}_{x t}(x, t)=\widetilde{z}_{x}(x, t) \widetilde{z}(x, t)^{-1} \widetilde{z}_{t}(x, t)+\widetilde{z}_{t}(x, t) \widetilde{z}(x, t)^{-1} \widetilde{z}_{x}(x, t) .
$$

Corollary 3.9. Assume that parameter matrices satisfy identity (3.19) and that

$$
\{0,1,-1\} \cap \sigma\left(A_{k}\right)=\emptyset \quad(k=1,2) .
$$

Let an invertible matrix function z satisfy main chiral field equation (3.30) and be two times continuously differentiable. Then the matrix function $\widetilde{z}$ given by (3.42) in the points of invertibility of $S$ also satifies main chiral field equation.

Our next examples deal with the construction of new (local) solutions of integrable elliptic sine-Gordon and sinh-Gordon equations from the initial solutions. See, for instance, $[10,36]$ and references therein for some related literature and auxiliary systems .

Example 3.10. Elliptic sine-Gordon equation

$$
v_{t t}+v_{x x}=\sin v \quad(v=\bar{v})
$$

is equivalent to the compatibility condition (2.45) of the auxiliary systems (3.16), where

$$
\begin{aligned}
G & =\frac{1}{4}\left(i \lambda \zeta+v_{t} j-\frac{i}{\lambda} J \zeta J\right), \quad \zeta=\left[\begin{array}{cc}
0 & e^{-i v / 2} \\
e^{i v / 2} & 0
\end{array}\right], \\
F & =-\frac{1}{4}\left(\lambda \zeta+v_{x} j+\frac{1}{\lambda} J \zeta J\right),
\end{aligned}
$$

and matrices $j$ and $J$ are defined in (1.9) after putting $p=1$. We put also

$$
A_{1}=A, \quad A_{2}=-\left(A^{*}\right)^{-1}, \quad \Pi_{1} \equiv \Pi, \quad \Pi_{2}(0,0)=A^{-1} \Pi(0,0) J .
$$

Thus, we have three parameter matrices, that is, $n \times n$ matrices $A$ and $S(0,0)$ and an $n \times m$ matrix $\Pi(0,0)$. We assume that $v$ satisfies (3.44), that $\operatorname{det} A \neq 0$ and $S(0,0)=S(0,0)^{*}$, and that there is a matrix $U$ such that equalities

$$
\bar{A}=U A^{-1} U^{-1}, \quad \bar{\Pi}(0,0)=U \Pi(0,0), \quad \bar{S}(0,0)=U A S(0,0) A^{*} U^{*}
$$

hold. Here $\bar{A}$ is the matrix with the entries, which are complex conjugate to the corresponding entries of A. By (3.47) the identity (3.19), which should be satisfied by the parameter matrices, takes the form

$$
A S(0,0) A^{*}+S(0,0)=\Pi(0,0) J \Pi(0,0)^{*} .
$$

Compare (3.17) and (3.18) with (3.45) and (3.46), respectively, to see that

$$
r=1, q_{1}=(-i / 4) \zeta, q_{0}=\left(-v_{t} / 4\right) j ; l=r_{1}=1, c_{1}=0, q_{11}=(i / 4) J \zeta J ;
$$




$$
R=1, Q_{1}=(1 / 4) \zeta, Q_{0}=\left(v_{x} / 4\right) j ; L=R_{1}=1, C_{1}=0, Q_{11}=(1 / 4) J \zeta J .
$$

Thus, in view of (3.47) equations (3.3) and (3.20) take the form

$$
\Pi_{x}=\frac{1}{4}\left(-i A \Pi \zeta-v_{t} \Pi j+i A^{-1} \Pi J \zeta J\right), \quad \Pi_{t}=\frac{1}{4}\left(A \Pi \zeta+v_{x} \Pi j+A^{-1} \Pi J \zeta J\right) .
$$

As $\bar{\zeta}=J \zeta J$ and $\bar{A}=U A^{-1} U^{-1}$, one can see that both $\Pi$ and $z=\overline{U \Pi}$ satisfy (3.50). According to $(3.48) \Pi(0,0)=\overline{U \Pi}(0,0))$, and so we derive

$$
\Pi(x, t) \equiv \overline{U \Pi}(x, t) .
$$

Equations (3.4) and (3.21), which define $\Pi_{2}^{*}$, take the form

$$
\begin{aligned}
\left(\Pi_{2}^{*}\right)_{x} & =\frac{1}{4}\left(i \zeta \Pi_{2}^{*} A_{2}+v_{t} j \Pi_{2}^{*}-i J \zeta J \Pi_{2}^{*} A_{2}^{-1}\right), \\
\left(\Pi_{2}^{*}\right)_{t} & =\frac{1}{4}\left(-\zeta \Pi_{2}^{*} A_{2}-v_{x} j \Pi_{2}^{*}-J \zeta J \Pi_{2}^{*} A_{2}^{-1}\right) .
\end{aligned}
$$

As $\zeta=\zeta^{*}$ and $A_{2}=-\left(A^{*}\right)^{-1}$, it follows from (3.50) that

$$
z(x, t)=A^{-1} \Pi(x, t) J
$$

satisfies equations (3.52) and (3.53) for $\Pi_{2}$. Moreover, we have $\Pi_{2}(0,0)=A^{-1} \Pi(0,0) J=z(0,0)$. In other words we have

$$
\Pi_{2}(x, t) \equiv A^{-1} \Pi(x, t) J
$$

and identity (3.26) takes the form

$$
A S(x, t) A^{*}+S(x, t)=\Pi(x, t) J \Pi(x, t)^{*} .
$$

By (3.5), (3.22), (3.54), and by the second equality in (3.47) the relations

$$
S_{x}=\frac{i}{4}\left(A^{-1} \Pi J \zeta \Pi^{*}-\Pi \zeta J \Pi^{*}\left(A^{*}\right)^{-1}\right), S_{t}=\frac{1}{4}\left(A^{-1} \Pi J \zeta \Pi^{*}+\Pi \zeta J \Pi^{*}\left(A^{*}\right)^{-1}\right)
$$

hold. Formulas (3.48), (3.51), and (3.55) imply $\bar{S}_{x}=U A S_{x} A^{*} U^{*}, \bar{S}_{t}=U A S_{t} A^{*} U^{*}$ and finally

$$
\bar{S} \equiv U A S A^{*} U^{*}
$$

It follows from (3.12), (3.51), (3.54), and (3.56) that

$$
X_{-1}=J \Pi^{*}\left(A^{*}\right)^{-1} S^{-1} A^{-1} \Pi=J \Pi^{*} U^{*}\left(U A S A^{*} U^{*}\right)^{-1} U \Pi=J \overline{\left(\Pi^{*} S^{-1} \Pi\right)} .
$$

According to (3.12), (3.15), and (3.24) we have

$$
Z(x, t):=w_{A}(x, t, 0)=I_{2}-X_{-1}, \quad Z(x, t)^{-1}=w_{A}(x, t, 0)^{-1}=I_{2}+Y_{-1} .
$$


Moreover, in view of (3.12), (3.54), and equality $\Pi^{*} S^{-1} \Pi=\left(\Pi^{*} S^{-1} \Pi\right)^{*}$ we get

$$
Y_{-1}=-J \Pi^{*} S^{-1} \Pi, \quad-\Pi^{*} S^{-1} \Pi=\left[\begin{array}{cc}
a & b \\
\bar{b} & d
\end{array}\right], \quad a=\bar{a}, \quad d=\bar{d} .
$$

Using (3.57)-(3.59) we derive

$$
Z Z^{-1}=\left[\begin{array}{cc}
1+b & d \\
a & 1+\bar{b}
\end{array}\right]\left[\begin{array}{cc}
1+\bar{b} & d \\
a & 1+b
\end{array}\right]=I_{2}
$$

If $1+b \neq 0$, formula (3.60) implies

$$
a=d=0, \quad|1+b|=1, \quad Z=\operatorname{diag}\{1+b, 1+\bar{b}\} .
$$

Put

$$
\widehat{v}=v+2 \arg (1+b), \quad \widehat{u}=Z^{-\frac{1}{2}} \widetilde{u}, \quad \widehat{G}=\widehat{u}_{x} \widehat{u}^{-1}, \quad \widehat{F}=\widehat{u}_{t} \widehat{u}^{-1},
$$

where $\widetilde{u}(x, t, \lambda)=w_{A}(x, t, \lambda) u(x, t, \lambda)$. In a way, which is similar to the proof of (3.29) in Theorem 3.7, we derive from (3.62) that

$$
\widehat{G}_{t}-\widehat{F}_{x}+[\widehat{G}, \widehat{F}]=0 .
$$

Moreover, one can see that $\widehat{G}$ and $\widehat{F}$ have the form (3.45) and (3.46), respectively, after one substitutes $\widehat{v}$ instead of $v$ into the right-hand sides of (3.45) and (3.46). Therefore, formula (3.63) implies that $\widehat{v}$ satisfies (3.44).

Corollary 3.11. Let an integer $n>0$ and matrices $A$ ( $\operatorname{det} A \neq 0), \Pi(0,0)$, and $S(0,0)=S(0,0)^{*}$ be fixed and satisfy conditions (3.48) and (3.49). Let $v$ satisfy elliptic sine-Gordon equation and be two times continuously differentiable. Then in the points, where $\operatorname{det} S \neq 0$ and $1+b \neq 0$, the function $\widehat{v}$ given by (3.62) satisfies elliptic sine-Gordon equation too.

If $\sigma(A) \cap \sigma\left(\left(-A^{*}\right)^{-1}\right)=\emptyset$, then the last equality in (3.48) follows from (3.49) and from the first two equalities in (3.48).

Remark 3.12. Using considerations from (3.58) one easily shows that a general equality $\left(I_{m}-\right.$ $\left.X_{-1}\right)\left(I_{m}+Y_{-1}\right)=I_{m}$ is true.

Elliptic sinh-Gordon equation

$$
v_{t t}+v_{x x}=\sinh v \quad(v=\bar{v})
$$

is equivalent to the compatibility condition (2.45) of the auxiliary systems (3.16), where

$$
\begin{aligned}
G & =-\frac{1}{4}\left(\lambda \zeta-i v_{t} j+\frac{1}{\lambda} \zeta^{*}\right), \quad \zeta=\left[\begin{array}{cc}
0 & e^{-v / 2} \\
e^{v / 2} & 0
\end{array}\right], \\
F & =-\frac{1}{4}\left(-i \lambda \zeta+i v_{x} j+\frac{i}{\lambda} \zeta^{*}\right) .
\end{aligned}
$$

Put

$$
A_{1}=A, \quad A_{2}=-\left(A^{*}\right)^{-1}, \quad \Pi_{1} \equiv \Pi, \quad \Pi_{2}(0,0)=A^{-1} \Pi(0,0) .
$$


Here we assume that

$$
\operatorname{det} A \neq 0, \quad S(0,0)=S(0,0)^{*},
$$

and that there is a matrix $U$ such that

$$
\bar{A}=U A^{-1} U^{-1}, \quad \bar{\Pi}(0,0)=U \Pi(0,0) J, \quad \bar{S}(0,0)=U A S(0,0) A^{*} U^{*} .
$$

Now, the identity (3.19) takes the form

$$
A S(0,0) A^{*}+S(0,0)=\Pi(0,0) \Pi(0,0)^{*} .
$$

Taking into account (3.65) and (3.66) introduce $\Pi$ by the equations

$$
\Pi_{x}=\frac{1}{4}\left(A \Pi \zeta-i v_{t} \Pi j+A^{-1} \Pi \zeta^{*}\right), \quad \Pi_{t}=\frac{1}{4}\left(-i A \Pi \zeta+i v_{x} \Pi j+i A^{-1} \Pi \zeta^{*}\right) .
$$

It is easy to see that $\Pi_{2} \equiv A^{-1} \Pi$, and so formulas (3.5) and (3.22) take the form

$$
S_{x}=\frac{1}{4}\left(\Pi \zeta \Pi^{*}\left(A^{*}\right)^{-1}+A^{-1} \Pi \zeta^{*} \Pi^{*}\right), S_{t}=\frac{i}{4}\left(A^{-1} \Pi \zeta^{*} \Pi^{*}-\Pi \zeta \Pi^{*}\left(A^{*}\right)^{-1}\right) .
$$

The matrix $Z=I_{2}-X_{-1}$ is again a diagonal matrix, and we have

$$
Z=I_{2}-\Pi^{*}\left(A^{*}\right)^{-1} S^{-1} A^{-1} \Pi=\operatorname{diag}\left\{Z_{11}, Z_{11}^{-1}\right\}, \quad Z_{11}=\overline{Z_{11}} .
$$

The proof of formula (3.73) is similar to the proof of (3.61) and the following corollary is proved in a quite similar way to Corollary 3.11 .

Corollary 3.13. Let an integer $n>0$ and matrices $A, \Pi(0,0)$, and $S(0,0)$ be fixed and satisfy conditions (3.68)-(3.70). Let $v$ satisfy elliptic sinh-Gordon equation and be two times continuously differentiable. Then in the points, where $\operatorname{det} S \neq 0$ and $Z_{11} \neq 0$, the function

$$
\widehat{v}=v+2 \ln \left|Z_{11}\right|
$$

satisfies elliptic sinh-Gordon equation too. Here $Z_{11}$ is given by (3.71)-(3.73).

\section{GBDT for radial Dirac equation}

Radial Dirac equation has the form

$$
\left(-i \sigma_{2} \frac{d}{d x}+\frac{\kappa}{x} \sigma_{1}+V(x)\right) y=\lambda y \quad(x>0),
$$

or equivalently

$$
\left(\frac{d}{d x}+\lambda q_{1}+q_{0}(x)\right) y(x, \lambda)=0 \quad(x>0),
$$


where $\kappa$ is an integer, $\sigma_{i}$ are Pauli matrices,

$$
\begin{gathered}
\sigma_{1}=\left[\begin{array}{ll}
0 & 1 \\
1 & 0
\end{array}\right], \quad \sigma_{2}=\left[\begin{array}{cc}
0 & -i \\
i & 0
\end{array}\right], \quad \sigma_{3}=\left[\begin{array}{cc}
1 & 0 \\
0 & -1
\end{array}\right], \\
V(x)=v_{e}(x) I_{2}+v_{a}(x) \sigma_{1}+v_{s}(x) \sigma_{3},
\end{gathered}
$$

$v_{e}, v_{a}$, and $v_{s}$ are real-valued functions, which are locally integrable on intervals $[0, l$ ) (here and further $l \in \mathbb{R}_{+}$),

$$
\begin{gathered}
q_{1}=-\breve{J}, \quad \breve{J}:=\left(-i \sigma_{2}\right)^{-1}=\left[\begin{array}{cc}
0 & 1 \\
-1 & 0
\end{array}\right] \\
q_{0}(x)=v_{*}(x) \sigma_{3}+\breve{J}\left(v_{e}(x) I_{2}+v_{s}(x) \sigma_{3}\right), \quad v_{*}(x)=\frac{\kappa}{x}+v_{a}(x) .
\end{gathered}
$$

Note that $v_{e}, v_{a}$, and $v_{s}$ represent the electrostatic potential, the anomalous magnetic moment, and the sum of the mass and of the scalar potential, respectively.

If we put $p=1$ in (1.9) we have $\sigma_{1}=J$ and $\sigma_{3}=j$. Recall that Dirac-type system of the form (2.1), (2.2) was treated in Subsection 2.1. The radial Dirac equation differs from the Dirac-type system. Its structure (as well as the structure of $V$ in this section) is somewhat different and it usually has singularity at $x=0$, which is of interest from the physical point of view. We consider equation (4.1) independently from the results in the Subsection 2.1.

The double commutation method was applied to (4.1) in an interesting paper by G. Teschl [80]. By this method S. Albeverio, R. Hryniv, and Ya. Mykytyuk [4] proved that 1 is added to $\varkappa$, when an eigenvalue is removed and that 1 is subtracted from $\varkappa$, when an eigenvalue is inserted. (In this section we actively use some of the results from [4].) We apply GBDT to the radial Dirac equation (4.1). In particular, we construct explicitly potentials and fundamental solutions for the equation (4.1) with $\varkappa>0$ starting from the trivial equation (i.e., from $q_{0}=0$ ). The case formally corresponds to the removal of the eigenvalues, the iterated double commutation formulas for the insertion of the eigenvalues are given in [80].

Fundamental solution $u$ in this section is a non-degenerate $2 \times 2$ solution of (4.1), we do not require $u$ and $\widetilde{u}$ to be normalized at $x=0$.

\subsection{Main result}

The following procedure to construct explicit solutions of the radial Dirac equation is an immediate corollary of Theorem 4.9 from Subsection 4.2.

Theorem 4.1. To construct a class of explicit solutions of equation (4.1) with some fixed integer $\kappa$, fix an integer $m>0$ and $m \times m$ matrices $\mathcal{A}_{1}$ and $\mathcal{S}_{1}>0$. Fix also a $\varkappa \times \varkappa$ lower triangular matrix $\mathcal{A}_{2}$ and an $(m+\varkappa) \times 2$ matrix $\Pi(0)=\operatorname{col}\left[\Psi_{1}(0) \quad \Psi_{2}(0)\right]$, where $\varkappa=|\kappa|$, col means column and $\Psi_{1}\left(\Psi_{2}\right)$ is an $m \times 2$ upper $(\varkappa \times 2$ lower $)$ block of $\Pi$. It is required that

$$
\mathcal{A}_{1} \mathcal{S}_{1}-\mathcal{S}_{1} \mathcal{A}_{1}^{*}=\Psi_{1}(0) \breve{J} \Psi_{1}(0)^{*}, \quad \Psi_{2}(0) \breve{J} \Psi_{2}(0)^{*}=0
$$


Moreover, for $h:=\left[\begin{array}{lllll}1 & 0 & 0 & \ldots & 0\end{array}\right] \Psi_{2}$ we assume that $h(0)=c\left[\begin{array}{ll}0 & 1\end{array}\right]$, when $\kappa$ is positive and odd or negative and even, and we assume that $h(0)=c\left[\begin{array}{ll}1 & 0\end{array}\right]$, when $\kappa$ is positive and even or negative and odd $(c \neq 0)$.

Introduce matrix $A$

$$
A:=\left[\begin{array}{cc}
\mathcal{A}_{1} & 0 \\
R & \mathcal{A}_{2}
\end{array}\right], \quad R=\Psi_{2}(0) \breve{J} \Psi_{1}(0)^{*} \mathcal{S}_{1}^{-1},
$$

and vectors $\theta_{1}, \theta_{2} \in \mathbb{C}^{m+\varkappa}$

$$
\left[\begin{array}{ll}
\theta_{1} & \theta_{2}
\end{array}\right]:=\Pi(0) \breve{K}, \quad \breve{K}:=\frac{1}{\sqrt{2}}\left[\begin{array}{ll}
1 & 1 \\
-i & i
\end{array}\right]
$$

Now, put

$$
\begin{aligned}
\Pi(x) & :=\left[\begin{array}{ll}
e^{i x A} \theta_{1} & e^{-i x A} \theta_{2}
\end{array}\right] \breve{K}^{*} \\
S(x) & :=\left[\begin{array}{ll}
\mathcal{S}_{1} & 0 \\
0 & 0
\end{array}\right]+\int_{0}^{x}\left(e^{i t A} \theta_{1} \theta_{1}^{*} e^{-i t A^{*}}+e^{-i t A} \theta_{2} \theta_{2}^{*} e^{i t A^{*}}\right) d t .
\end{aligned}
$$

Then, if $S(x)>0$ for $x>0$, the potential

$$
\widetilde{q}_{0}(x):=\breve{J} X(x) \breve{J}^{*}-X(x), \quad X=\left\{X_{i j}\right\}_{i, j=1}^{2}:=\Pi^{*} S^{-1} \Pi
$$

admits representation

$$
\widetilde{q}_{0}(x)=\frac{\kappa}{x} \sigma_{3}+\Upsilon(x)=\frac{\kappa}{x} \sigma_{3}+\widetilde{v}_{a}(x) \sigma_{3}-\widetilde{v}_{s}(x) \sigma_{1},
$$

where $\Upsilon, \widetilde{v}_{a}$, and $\widetilde{v}_{s}$ are bounded in the neighborhood of zero, and

$$
\widetilde{v}_{a}(x)=X_{22}(x)-X_{11}(x)-\frac{\kappa}{x}, \quad \widetilde{v}_{s}(x)=X_{12}(x)+X_{21}(x) .
$$

The fundamental solution $\widetilde{u}(x, \lambda)$ of system

$$
\left(\frac{d}{d x}+\lambda q_{1}+\widetilde{q}_{0}(x)\right) \widetilde{u}(x, \lambda)=0 \quad(x>0),
$$

is given by the formula

$$
\widetilde{u}(x, \lambda)=w_{A}(x, \lambda) \breve{K} \exp \left(-i \lambda x \sigma_{3}\right)
$$

where

$$
w_{A}(\lambda)=I_{2}-\breve{J} \Pi^{*} S^{-1}\left(A-\lambda I_{n}\right)^{-1} \Pi .
$$

By the neighbourhood of zero we mean the neighbourhood of the form $(0, \varepsilon)$ or $[0, \varepsilon)$. Note that if $S(x)>0$ for all $x \in(0, \varepsilon)$, then $S(x)>0$ for all $x \in \mathbb{R}_{+}$, the formulas in Theorem 4.1 are well-defined on $\mathbb{R}_{+}$, and $\widetilde{q}_{0}$ is infinitely differentiable on $\mathbb{R}_{+}$. The integer $m$ denotes in this section the order of the blocks $\mathcal{A}_{1}$ and $\mathcal{S}_{1}$ of $A$ and $S$, respectively. 


\subsection{Superposition of Darboux transformations}

In this subsection we apply GBDT to the radial Dirac equation. We factorize also the Darboux matrix, so that GBDT can be considered as a superposition of two other GBDTs. Finally, we formulate a general Theorem 4.9.

In view of (4.2), (4.5), and (4.6) we get

$$
r=1, \quad q_{1}=-\breve{J} ; \quad q_{k}^{*}=-\breve{J} q_{k} \breve{J}^{-1}, \quad k=0,1, \quad \breve{J}^{-1}=\breve{J}^{*}=-\breve{J} .
$$

Here we fix $n>0$ and parameter matrices $A_{1}=A, \Pi\left(x_{0}\right)$, and $S\left(x_{0}\right)=S\left(x_{0}\right)^{*}$. Formula (3.3) for $\Pi_{1}=\Pi$ takes the form

$$
\Pi_{x}=A \Pi q_{1}+\Pi q_{0}
$$

Putting

$$
A_{2}=A^{*}, \quad \Pi_{2}^{*}=\breve{J} \Pi^{*}
$$

and taking into account equation (4.19) and the third equalities in (4.18), we see that equation (3.4) for $\Pi_{2}^{*}$ is satisfied. By (4.18)-(4.20) formula (3.5) takes the form

$$
S_{x}=\Pi^{*} .
$$

Thus, we have $S(x)=S(x)^{*}$. It is required that

$$
A S\left(x_{0}\right)-S\left(x_{0}\right) A^{*}=\Pi\left(x_{0}\right) \breve{J} \Pi\left(x_{0}\right)^{*}
$$

for some $0<x_{0}<l$. The identity

$$
A S(x)-S(x) A^{*}=\Pi(x) \breve{J} \Pi(x)^{*}
$$

follows from (4.19), (4.21), and (4.22).

Remark 4.2. If $\Pi$ can be continuously extended to $\Pi(0)$, then $S$ can also be continuously extended to $S(0)$. So, in that case we consider $\Pi$ and $S$ defined on $[0, l)$, and for (4.23) to be true it suffices that (4.22) holds for $x_{0}=0$.

The next Corollary of Theorem 3.1 and of formula (3.14) is immediate

Corollary 4.3. Let relations (4.19), (4.21), and (4.22) hold. Then, in the points of the invertibility of $S(x)(x>0)$, we have

$$
\left(S(x)^{-1} \Pi(x)\right)_{x}(x)=A^{*} S(x)^{-1} \Pi(x) q_{1}+S(x)^{-1} \Pi(x) \widetilde{q}_{0}(x),
$$

where

$$
\widetilde{q}_{0}:=q_{0}+\breve{J} X \breve{J}^{*}-X, \quad X:=\breve{J}^{-1} X_{0}=\Pi^{*} S^{-1} \Pi .
$$

Moreover, the matrix function $w_{A}$ given by (4.17) satisfies the equation

$$
\frac{d}{d x} w_{A}(x, \lambda)=\widetilde{G}(x, \lambda) w_{A}(x, \lambda)-w_{A}(x, \lambda) G(x, \lambda),
$$




$$
\widetilde{G}(x, \lambda)=-\lambda q_{1}-\widetilde{q}_{0}(x), \quad G(x, \lambda)=-\lambda q_{1}-q_{0}(x) .
$$

In other words, we have

$$
\frac{d}{d x} \widetilde{u}(x, \lambda)=\widetilde{G}(x, \lambda) \widetilde{u}(x, \lambda),
$$

where

$$
\widetilde{u}(x, \lambda)=w_{A}(x, \lambda) u(x, \lambda) .
$$

Recall that by Remark $3.2 \widetilde{q}_{1}=q_{1}$. This explains why we have the coefficient $-q_{1}$ in the expression for $\widetilde{G}$ in (4.27). Equation (4.28) follows from (4.2) and (4.26). This equation is a GBDT transformation of the equation (4.2) and has the same structure. Namely, to get $\widetilde{q}_{0}$ instead of $q_{0}$ one substitutes into (4.6) $\widetilde{v}_{*}$ and $\widetilde{v}_{s}$ instead of $v_{*}$ and $v_{s}$, respectively. That is, by (4.6) and (4.25) we have

$$
\widetilde{q}_{0}(x)=\widetilde{v}_{e}(x) \breve{J}+v_{*}(x) \sigma_{3}-\widetilde{v}_{s}(x) \sigma_{1},
$$

where $\widetilde{v}_{e}(x)=v_{e}(x)$,

$$
\widetilde{v}_{*}(x)=v_{*}(x)+X_{22}(x)-X_{11}(x), \quad \widetilde{v}_{s}(x)=v_{s}(x)+X_{12}(x)+X_{21}(x),
$$

and $X_{k j}$ are the entries of $X$.

Remark 4.4. When $n=1$ and $A \in \mathbb{R}$ our transformation coincides with the double commutation transformation for Dirac equations treated in $[4,80]$. When $A \notin \mathbb{R}$ our transformation somewhat differs from the transformation in [4], because the transformation in [4] uses the equivalent (for $n=1)$ of $\Pi$ and of the transposition of $\Pi$, whereas we use here $\Pi$ and $\Pi^{*}$, so that $\widetilde{v}_{a}$ and $\widetilde{v}_{s}$ are real valued.

We will be interested in the transformation of the equation (4.2) with $\kappa=0$ into equation with integer nonzero $\kappa$.

Example 4.5. Let $n=1, q_{0} \equiv 0, S(0)=0$. Notice that we have

$$
q_{1}=-\breve{J}=i \breve{K} \sigma_{3} \breve{K}^{*}, \quad \breve{K}:=\frac{1}{\sqrt{2}}\left[\begin{array}{ll}
1 & 1 \\
-i & i
\end{array}\right], \quad \breve{K}^{*}=\breve{K}^{-1}
$$

and put $(\Pi \breve{K})(0)=\left[\begin{array}{ll}1 & \alpha\end{array}\right]$. Taking into account $q_{0} \equiv 0$ and (4.32), we rewrite (4.19) and (4.21) in the form

$$
(\Pi \breve{K})_{x}=i A \Pi \breve{K} \sigma_{1}, \quad S_{x}=(\Pi \breve{K})(\Pi \breve{K})^{*}
$$

It is immediate from (4.33) that

$$
\Pi(x) \breve{K}=\left[\begin{array}{ll}
e^{i x A} & \alpha e^{-i x A}
\end{array}\right] .
$$

Recall that $S(0)=0$. Thus, when $A \neq \bar{A}$, we require additionally $|\alpha|=1$ so that (4.22) holds for $x_{0}=0$. Hence, formula (4.23) is true for all $x>0$ (see Remark 4.2). Using (4.23), (4.33), and (4.34) we get

$$
\begin{aligned}
& S(x)=i(A-\bar{A})^{-1}\left(e^{i x(\bar{A}-A)}-e^{i x(A-\bar{A})}\right) \text { for } A \neq \bar{A}, \\
& S(x)=\left(1+|\alpha|^{2}\right) x \quad \text { for } \quad A \in \mathbb{R} .
\end{aligned}
$$


By (4.31), (4.34), and (4.35) we have for $A \neq \bar{A}$ the equality

$$
\widetilde{v}_{*}(x)=i(A-\bar{A})\left(\alpha e^{-i x(A+\bar{A})}+\bar{\alpha} e^{i x(A+\bar{A})}\right)\left(e^{i x(\bar{A}-A)}-e^{i x(A-\bar{A})}\right)^{-1} .
$$

In a similar way we have

$$
\widetilde{v}_{*}(x)=-\left(\alpha e^{-2 i x A}+\bar{\alpha} e^{2 i x A}\right)\left(\left(1+|\alpha|^{2}\right) x\right)^{-1} \quad \text { for } \quad A \in \mathbb{R} .
$$

From (4.36) and (4.37) we derive that

$$
\widetilde{v}_{*}(x)=\frac{\widetilde{\kappa}}{x}+\widetilde{v}_{a}(x), \quad \widetilde{\kappa}=-\frac{\alpha+\bar{\alpha}}{1+|\alpha|^{2}},
$$

where $\widetilde{v}_{a}$ is continuous on $[0, \infty)$. When $\alpha= \pm 1$, we obtain $\widetilde{\kappa}=\mp 1$, that is, $\kappa$ is an integer.

To study GBDT we will need, in particular, to split it into a superposition of several transformations, and our next result is dedicated to this procedure. Let $A$ be a block lower triangular matrix:

$$
A=\left[\begin{array}{rr}
A_{11} & 0 \\
A_{21} & A_{22}
\end{array}\right], \quad S=\left[\begin{array}{ll}
S_{11} & S_{12} \\
S_{21} & S_{22}
\end{array}\right]
$$

where $A_{11}$ and $S_{11}$ are $n_{1} \times n_{1}$ matrices, $A_{22}$ and $S_{22}$ are $n_{2} \times n_{2}$ matrices, $n=n_{1}+n_{2}$. In our further considerations we fix some value of $x$ and omit temporarily the variable $x$ in the notations. Assume det $S \neq 0$ and det $S_{11} \neq 0$, and denote by $T_{22}$ the $n_{2} \times n_{2}$ right lower block of $T=S^{-1}$. The invertibility of $T_{22}$ follows from the invertibility of $S_{11}$ and $S$. One can check directly that

$$
T=\left[\begin{array}{cc}
S_{11}^{-1}+S_{11}^{-1} S_{12} T_{22} S_{21} S_{11}^{-1} & -S_{11}^{-1} S_{12} T_{22} \\
-T_{22} S_{21} S_{11}^{-1} & T_{22}
\end{array}\right], \quad T_{22}^{-1}=S_{22}-S_{21} S_{11}^{-1} S_{12} .
$$

As $A$ is a block lower triangular matrix and $\operatorname{det} S \neq 0, \operatorname{det} S_{11} \neq 0$, so $w_{A}$ admits factorisation $[76,77]$

$$
\begin{gathered}
w_{A}(\lambda)=w_{2}(\lambda) w_{1}(\lambda), \\
w_{1}(\lambda)=I_{2}-\breve{J} \Pi^{*} P_{1}^{*} S_{11}^{-1}\left(A_{11}-\lambda I_{n_{1}}\right)^{-1} P_{1} \Pi \\
w_{2}(\lambda)=I-\breve{J} \Pi^{*} S^{-1} P_{2}^{*}\left(A_{22}-\lambda I_{n_{2}}\right)^{-1} T_{22}^{-1} P_{2} S^{-1} \Pi,
\end{gathered}
$$

where

$$
P_{1}=\left[\begin{array}{ll}
I_{n_{1}} & 0
\end{array}\right], \quad P_{2}=\left[\begin{array}{ll}
0 & I_{n_{2}}
\end{array}\right] .
$$

One can easily see that by (4.39) we have $P_{1} A=A_{11} P_{1}$ and $A P_{2}^{*}=P_{2}^{*} A_{22}$. Hence, the identity

$$
A_{11} S_{11}-S_{11} A_{11}^{*}=\pi_{1} \breve{J} \pi_{1}^{*}, \quad \pi_{1}:=P_{1} \Pi
$$

follows from (4.23). Now, compare (4.17) and (4.42) to see that $w_{1}=w_{A_{11}}$. That is, we get $w_{1}$ after substitution of $A_{11}$ instead of $A$, of $S_{11}$ instead of $S$ and of $\pi_{1}$ instead of $\Pi$ into (4.17). Identity (4.45) is the equivalent of (4.23) written for the new transfer matrix function $w_{A_{11}}$. 
Moreover, from (4.23) it follows that $T A-A^{*} T=T \Pi \breve{J} \Pi^{*} T$. Therefore, we derive $T_{22} A_{22}-$ $A_{22}^{*} T_{22}=P_{2} T \Pi \breve{J} \Pi^{*} T P_{2}^{*}$ or, equivalently

$$
A_{22} T_{22}^{-1}-T_{22}^{-1} A_{22}^{*}=\pi_{2} \breve{J} \pi_{2}^{*}, \quad \pi_{2}=T_{22}^{-1} P_{2} S^{-1} \Pi .
$$

Compare (4.17) and (4.43) to see that $w_{2}=w_{A_{22}}$, where $T_{22}^{-1}$ is substituted into (4.17) instead of $S$ and $\pi_{2}$ is substituted instead of $\Pi$.

Remark 4.6. Corollary 4.3 defines by formulae (4.25) and (4.29) GBDT transformation of the coefficient $q_{0}$ and solutions $u$ of the radial Dirac equation (4.2). The next proposition shows that the GBDT generated by the parameter matrices $A, S\left(x_{0}\right)$ and $\Pi\left(x_{0}\right)$ can be treated as a superposition of two transformations, i.e., GBDTs generated by $A_{11}, S_{11}\left(x_{0}\right), \pi_{1}\left(x_{0}\right)$ and $A_{22}, T_{22}\left(x_{0}\right)^{-1}, \pi_{2}\left(x_{0}\right)$, respectively. In particular, our next proposition shows that $\pi_{1}$ and $S_{11}$ satisfy analogs of (4.19) and (4.21). The same is true for $T_{22}^{-1}$ and $\pi_{2}$. According to Corollary 4.3 it means that $w_{1}(x, \lambda)$ and $w_{2}(x, \lambda)$ are Darboux matrices.

Proposition 4.7. Let relations (4.19), (4.21), and (4.22) be valid and let A be a block lower triangular matrix. Then, in the points of the invertibility of $S(x)$ and $S_{11}(x)(x>0)$, we have

$$
\begin{aligned}
& \left(S_{11}\right)_{x}=\pi_{1} \pi_{1}^{*}, \quad\left(\pi_{1}\right)_{x}=A_{11} \pi_{1} q_{1}+\pi_{1} q_{0} . \\
& \left(T_{22}^{-1}\right)_{x}=\pi_{2} \pi_{2}^{*}, \quad\left(\pi_{2}\right)_{x}=A_{22} \pi_{2} q_{1}+\pi_{2} \widehat{q}_{0},
\end{aligned}
$$

where $\pi_{1}$ and $\pi_{2}$ are given by (4.45) and (4.46), respectively, and

$$
\widehat{q}_{0}=q_{0}+\breve{J} \widehat{X} \breve{J}^{*}-\widehat{X}, \quad \widehat{X}=\pi_{1}^{*} S_{11}^{-1} \pi_{1} .
$$

Proof. Multiply from the left both sides of (4.19) by $P_{1}$ and use $P_{1} A=A_{11} P_{1}$ to get the second relation in (4.47). Multiply by $P_{1}$ from the left and by $P_{1}^{*}$ from the right both sides of (4.21) to get the first relation in (4.47). In view of (4.21) and of the definition of $\pi_{2}$ in (4.46), we have also the first relation in (4.48):

$$
\begin{aligned}
& \frac{d}{d x} T_{22}^{-1}=-T_{22}^{-1}\left(\frac{d}{d x} T_{22}\right) T_{22}^{-1}=-T_{22}^{-1} P_{2}\left(\frac{d}{d x} T\right) P_{2}^{*} T_{22}^{-1} \\
& =T_{22}^{-1} P_{2} S^{-1} \Pi \Pi^{*} S^{-1} P_{2}^{*} T_{22}^{-1}=\pi_{2} \pi_{2}^{*}
\end{aligned}
$$

Now, use (4.24), (4.50), and equality $P_{2} A^{*}=A_{22}^{*} P_{2}$ to differentiate $\pi_{2}$ :

$$
\begin{aligned}
& \left(\pi_{2}\right)_{x}=\pi_{2} \pi_{2}^{*} P_{2} S^{-1} \Pi+T_{22}^{-1} P_{2}\left(A^{*} S^{-1} \Pi q_{1}+S^{-1} \Pi \widetilde{q}_{0}\right) \\
& =\pi_{2} \pi_{2}^{*} P_{2} S^{-1} \Pi+T_{22}^{-1} P_{2} S^{-1} \Pi \widetilde{q}_{0}+T_{22}^{-1} A_{22}^{*} P_{2} S^{-1} \Pi q_{1} .
\end{aligned}
$$

Taking into account both relations in (4.46), both relations in (4.25), and $q_{1}=\breve{J}^{*}$, rewrite (4.51) in the form

$$
\begin{aligned}
& \left(\pi_{2}\right)_{x}=\pi_{2} \pi_{2}^{*} P_{2} S^{-1} \Pi+\pi_{2} \widetilde{q}_{0}+A_{22} T_{22}^{-1} P_{2} S^{-1} \Pi q_{1} \\
& -\pi_{2} \pi_{2}^{*} P_{2} S^{-1} \Pi q_{1}=A_{22} \pi_{2} q_{1}+\pi_{2}\left(q_{0}+\breve{J} \Pi^{*} S^{-1} \Pi \breve{J}^{*}\right. \\
& \left.-\Pi^{*} S^{-1} \Pi+\pi_{2}^{*} P_{2} S^{-1} \Pi-\breve{J} \pi_{2}^{*} P_{2} S^{-1} \Pi \breve{J}^{*}\right) .
\end{aligned}
$$


Finally, note that according to the definition of $\pi_{2}^{*}$ and formula (4.40) we get

$$
\Pi^{*}-\pi_{2}^{*} P_{2}=\Pi^{*}\left(I_{n}+\left[\begin{array}{c}
S_{11}^{-1} S_{12} \\
-I_{n_{2}}
\end{array}\right] P_{2}\right)=\pi_{1}^{*} S_{11}^{-1}\left[\begin{array}{ll}
S_{11} & S_{12}
\end{array}\right] .
$$

It follows that

$$
\left(\Pi^{*}-\pi_{2}^{*} P_{2}\right) S^{-1} \Pi=\pi_{1}^{*} S_{11}^{-1} \pi_{1} .
$$

Substitute (4.54) into (4.52) to derive

$$
\left(\pi_{2}\right)_{x}=A_{22} \pi_{2} q_{1}+\pi_{2}\left(q_{0}+\breve{J} \pi_{1}^{*} S_{11}^{-1} \pi_{1} \breve{J}^{*}-\pi_{1}^{*} S_{11}^{-1} \pi_{1}\right) .
$$

Formulas (4.49) and (4.55) imply the second relation in (4.48).

Proposition 4.7 deals with the $S$-nodes, which appear in the process of factorization (4.41) of the transfer matrix function $w_{A}$. An inverse in a certain sense result is given in the next proposition.

Proposition 4.8. Let $w_{\mathcal{A}_{j}}(j=1,2)$ be transfer matrix functions of the form (4.17) corresponding to the $S$-nodes $\mathcal{A}_{j}, \mathcal{S}_{j}$ and $\Psi_{j}$, where $\mathcal{A}_{1}$ and $\mathcal{S}_{1}$ are $m \times m$ matrices, $\mathcal{A}_{2}$ and $\mathcal{S}_{2}$ are $\varkappa \times \varkappa$ matrices, $\Psi_{1}$ is an $m \times 2$ matrix, $\Psi_{2}$ is a $\varkappa \times 2$ matrix. Then we have $w_{\mathcal{A}_{2}}(\lambda) w_{\mathcal{A}_{1}}(\lambda)=w_{A}(\lambda)$, where $w_{A}$ is the transfer matrix function corresponding to the $S$-node of the form

$$
A=\left[\begin{array}{cc}
\mathcal{A}_{1} & 0 \\
R & \mathcal{A}_{2}
\end{array}\right], \quad S=\left[\begin{array}{ll}
\mathcal{S}_{1} & 0 \\
0 & \mathcal{S}_{2}
\end{array}\right], \quad \Pi=\left[\begin{array}{c}
\Psi_{1} \\
\Psi_{2}
\end{array}\right] ; \quad R=\Psi_{2} \breve{J} \Psi_{1}^{*} \mathcal{S}_{1}^{-1} .
$$

Proof. From $\mathcal{A}_{j} \mathcal{S}_{j}-\mathcal{S}_{j} \mathcal{A}_{j}^{*}=\Psi_{j} \breve{J} \Psi_{j}^{*}(p=1,2)$ and representation (4.56), where $R=\Psi_{2} \breve{J} \Psi_{1}^{*} \mathcal{S}_{1}^{-1}$, it follows that $A S-S A^{*}=\Pi \breve{J} \Pi^{*}$. Now, from (4.41), (4.42), and (4.56) we have $w_{A}(\lambda)=$ $w_{2}(\lambda) w_{\mathcal{A}_{1}}(\lambda)$. By (4.40), (4.43), (4.46), and (4.56) we obtain $T_{22}^{-1}=\mathcal{S}_{2}, \pi_{2}=\Psi_{2}$ and, finally, $w_{2}=w_{\mathcal{A}_{2}}$.

Using Corollary 4.3 and Proposition 4.7 we prove in the next section the following theorem.

Theorem 4.9. Let the initial system (4.2), where the coefficient $q_{0}(x)$ is bounded in the neighbourhood of zero, be given. Let the parameter matrices $A, S(0)$, and $\Pi(0)$ have the block form

$$
A=\left[\begin{array}{cc}
\mathcal{A}_{1} & 0 \\
R & \mathcal{A}_{2}
\end{array}\right], \quad S(0)=\left[\begin{array}{ll}
\mathcal{S}_{1} & 0 \\
0 & 0
\end{array}\right], \quad \Pi(0)=\left[\begin{array}{l}
\Psi_{1}(0) \\
\Psi_{2}(0)
\end{array}\right],
$$

where $\mathcal{A}_{1}$ and $\mathcal{S}_{1}$ are $m \times m$ matrices, $A$ and $S(0)$ are $(m+\varkappa) \times(m+\varkappa)$ matrices $(\varkappa>0)$, $\Psi_{1}(0)$ is an $m \times 2$ matrix, $\Psi_{2}(0)$ is a $\varkappa \times 2$ matrix, $\mathcal{A}_{2}$ is a lower triangular $\varkappa \times \varkappa$ matrix, and $R=\Psi_{2}(0) \breve{J} \Psi_{1}(0)^{*} \mathcal{S}_{1}^{-1}$. Let the relations

$$
\mathcal{A}_{1} \mathcal{S}_{1}-\mathcal{S}_{1} \mathcal{A}_{1}^{*}=\Psi_{1}(0) \breve{J} \Psi_{1}(0)^{*}, \quad \Psi_{2}(0) \breve{J} \Psi_{2}(0)^{*}=0, \quad \mathcal{S}_{1}>0
$$

hold. Introduce $\Pi(x)$ and $S(x)$ by (4.19) and (4.21), respectively, and assume $S(x)>0$ for $x>0$. Put $h:=\left[\begin{array}{lllll}1 & 0 & 0 & \ldots & 0\end{array}\right] \Psi_{2}$ and suppose

$$
h(0)=c\left[\begin{array}{ll}
1 & 0
\end{array}\right] \quad \text { or } \quad h(0)=c\left[\begin{array}{ll}
0 & 1
\end{array}\right] \quad(c \neq 0) .
$$


Then the transformed system

$$
\left(\frac{d}{d x}+\lambda q_{1}+\widetilde{q}_{0}(x)\right) \widetilde{u}(x, \lambda)=0
$$

where $\widetilde{q}_{0}$ is given by (4.25), has the fundamental solution $\widetilde{u}=w_{A} u$. Moreover, the coefficient $\widetilde{q}_{0}$ admits representation

$$
\widetilde{q}_{0}(x)=\frac{\kappa}{x} \sigma_{3}+\Upsilon(x),
$$

where $\Upsilon$ is bounded in the neighbourhood of zero. Here, $\kappa=\varkappa$, if $\varkappa$ is odd and $h(0)=c\left[\begin{array}{ll}0 & 1\end{array}\right]$ or if $\varkappa$ is even and $h(0)=c\left[\begin{array}{ll}1 & 0\end{array}\right]$. We have $k=-\varkappa$, if $\varkappa$ is even and $h(0)=c\left[\begin{array}{ll}0 & 1\end{array}\right]$ or if $\varkappa$ is odd and $h(0)=c[1 \quad 0]$.

\subsection{Proof of Theorem 4.9}

When $\Pi(x)$ is squarely integrable in the neighbourhood of 0 , using (4.21) we get $S(x)=S(0)+$ $\int_{0}^{x} \Pi(t) \Pi(t)^{*} d t$. When $S(0)=0$, we have

$$
S(x)=\int_{0}^{x} \Pi(t) \Pi(t)^{*} d t
$$

Lemma 4.10. Let $n=1, A \in \mathbb{C}, S(0)=0$, and $\Pi(0)=\alpha_{1}\left[\begin{array}{ll}1 & \alpha_{2}\end{array}\right] \breve{K}^{*}$, where $\alpha_{1} \neq 0$ and $\alpha_{2}= \pm 1$. Assume that the potential $q_{0}$ of the initial system (4.2) is

(i) bounded on $(0, \varepsilon)$ for some $\varepsilon>0$

or

(ii) has a bounded limit, when $x$ tends to +0 .

Then for the GBDT tranformation of $q_{0}$ defined by (4.25) we have

$$
\widetilde{q}_{0}(x)=\Upsilon_{ \pm}(x) \mp \frac{1}{x} \sigma_{3},
$$

where $\Upsilon_{+}(x)$ and $\Upsilon_{-}(x)$ are bounded in the neighbourhood of zero, if (i) is fulfilled, and have a bounded limit, when $x$ tends to +0 , if (ii) is true.

Proof. Note that under conditions of the lemma we have $A S(0)-S(0) A^{*}=0$. According to (4.32) we have $\breve{K}^{*} \breve{J} \breve{K}=-i \sigma_{3}$, and so $\Pi(0) \breve{J} \Pi(0)^{*}=0$. Then the identity (4.23) holds for $x=0$, and therefore it is satisfied for all $x \geq 0$. Hence, the matrix function $\widetilde{q}_{0}$ given by (4.25) is the GBDT transformation of $q_{0}$.

By the definition of $\breve{K}$ in (4.32) we get

$$
\Pi(0)=c[1 \quad 0] \text { for } \alpha_{2}=1, \quad \Pi(0)=c\left[\begin{array}{ll}
0 & 1
\end{array}\right] \text { for } \alpha_{2}=-1 \quad(c \neq 0) .
$$

By (4.19) one can see that

$$
\Pi(x)-\Pi(0)=x f(x)
$$


where $f$ is bounded on $[0, \varepsilon)$, if $q_{0}$ is bounded, and $f$ has a bounded limit for $x \rightarrow+0$, if $q_{0}$ has a bounded limit. From (4.61), (4.63), and (4.64) it follows that

$$
S(x)=|c|^{2} x+x^{2} f_{1}(x),
$$

where $f_{1}$ is also bounded on $[0, \varepsilon)$, if $q_{0}$ is bounded, and $f_{1}$ has a bounded limit for $x \rightarrow+0$, if $q_{0}$ has a bounded limit. Taking into account (4.25) and (4.63)-(4.65) we obtain the statement of the lemma.

Recall the notations from Proposition 4.7 and consider the case $n>1, n_{1}=1, n_{2}=n-1$, so that $\pi_{1}$ is the first block (i.e. the first row) of $\Pi$, and $\pi_{2}$ is given by (4.46).

Lemma 4.11. Let the initial system (4.2) be given and let the potential $q_{0}$ be bounded in the neighbourhood of zero. Suppose that $A$ is a lower triangular $n \times n(n>1)$ matrix, that relations (4.19) and (4.21) hold and that

$$
S(0)=0, \quad \Pi(0) \breve{J} \Pi(0)^{*}=0, \quad S(x)>0 \quad \text { for } \quad x>0 .
$$

Then, we have

$$
\begin{aligned}
& \widehat{q}_{0}(x)=\widehat{\Upsilon}_{+}(x)-\frac{1}{x} \sigma_{3} \quad \text { for } \quad \pi_{1}(0)=c\left[\begin{array}{ll}
1 & 0
\end{array}\right] ; \\
& \widehat{q}_{0}(x)=\widehat{\Upsilon}_{-}(x)+\frac{1}{x} \sigma_{3} \text { for } \pi_{1}(0)=c\left[\begin{array}{ll}
0 & 1
\end{array}\right] \text {, }
\end{aligned}
$$

where $c \in \mathbb{C} \backslash\{0\}, \widehat{q}_{0}$ is given by (4.49) and $\widehat{\Upsilon}_{ \pm}$is bounded in the neighbourhood of zero. Moreover, the matrix function $\pi_{2}(x)$ is continuous at zero and the relations

$$
\lim _{x \rightarrow+0}\left(T_{22}^{-1}\right)(x)=0, \quad\left(T_{22}^{-1}\right)(x)>0 \quad \text { for } \quad x>0, \quad \pi_{2}(0) \breve{J} \pi_{2}(0)^{*}=0
$$

hold for both cases (4.67) and (4.68). The matrix functions $T_{22}^{-1}$ and $\pi_{2}$ satisfy (4.48).

Proof. First, compare Proposition 4.7 and Lemma 4.10 to see that $\widehat{q}_{0}$ from (4.49) coincides with $\widetilde{q}_{0}$ from (4.62). Thus, (4.67) and (4.68) are immedate from Lemma 4.10.

As $S(x)>0$, so we get $T(x)>0, T_{22}(x)>0$ and, finally, $\left(T_{22}^{-1}\right)(x)>0$ (i.e., the second relation in (4.69) holds). Similar to the case treated in Lemma 4.10 we have $A S(0)-S(0) A^{*}=0$ and $\Pi(0) \breve{J} \Pi(0)^{*}=0$, that is, $(4.23)$ holds at $x=0$. Hence, the conditions of Proposition 4.7 hold. By Proposition 4.7, $T_{22}^{-1}$ and $\pi_{2}$ satisfy (4.48).

Recall that $S(0)=0$. Therefore, using formulae (4.61) and (4.65) we derive

$$
S_{22}(0)=0, \quad \lim _{x \rightarrow+0} S_{21}(x) S_{11}(x)^{-1} S_{12}(x)=0 .
$$

The second equality in (4.40) and formula (4.70) imply the first relation in (4.69). From the definition of $\pi_{2}$ in (4.46) and from the first relation in (4.40) it follows that

$$
\pi_{2}=\left[-S_{21} S_{11}^{-1} \quad I_{n-1}\right] \Pi .
$$

Now, use again (4.61) and (4.65) to see that $\pi_{2}$ has a limit, when $x$ tends to +0 . Therefore, the last relation in (4.69) is immediate from the first relations in (4.46) and (4.69). 
Next, consider the case of the initial system with $\kappa \neq 0$, where $V \in L_{2 \times 2}^{p}(0, \varepsilon)$, that is, the entries of $V$ belong to $L^{p}$ in the neighbourhood of zero.

Lemma 4.12. Let the initial Dirac system (4.2) be given, where

$$
q_{0}(x)=i \sigma_{2}\left(V(x)+\frac{\kappa}{x} \sigma_{1}\right)=i \sigma_{2} V(x)+\frac{\kappa}{x} \sigma_{3}, \quad \kappa \neq 0,
$$

and $V \in L_{2 \times 2}^{p}(0, \varepsilon)$. Suppose that $A$ is a lower triangular $n \times n(n>1)$ matrix, that relations (4.19) and (4.21) hold, that $S$ and $\Pi$ are continuous at zero and that (4.66) is true. Then, putting $n_{1}=1$, we get

$$
\widehat{q}_{0}(x)=-\frac{1+\kappa}{x} \sigma_{3}+\widehat{\Upsilon}_{+}(x) \text { for } \kappa>0, \quad \widehat{q}_{0}(x)=\frac{1-\kappa}{x} \sigma_{3}+\widehat{\Upsilon}_{-}(x) \text { for } \kappa<0,
$$

where $\widehat{q}_{0}$ is given by (4.49) and $\widehat{\Upsilon}_{ \pm} \in L_{2 \times 2}^{p}$ in the neighbourhood of zero. Moreover, the matrix function $\pi_{2}(x)$ is continuous at zero and the relations (4.69) hold. The matrix functions $T_{22}^{-1}$ and $\pi_{2}$ satisfy (4.48).

To prove this lemma we shall need Lemma A1 from [4]:

Lemma 4.13. Let $q_{0}$ be given by (4.72). Assume $\kappa \in \mathbb{Z} \backslash 0, \lambda \in \mathbb{C}$, and $V \in L_{2 \times 2}^{p}(1 \leq p<\infty)$ in the neighbourhood of zero. Then, there are two types of nontrivial solutions $y=\operatorname{col}\left[\begin{array}{ll}y_{1} & y_{2}\end{array}\right]$ of (4.2). Namely, as $x \rightarrow+0$, either the limit $\lim _{x \rightarrow+0} x^{-\kappa} y(x, \lambda) \neq 0$ exists and $x^{-\kappa-1} y_{1}(x, \lambda) \in L^{p}$ in the neighbourhood of zero or the limit $\lim _{x \rightarrow+0} x^{\kappa} y(x, \lambda) \neq 0$ exists and $x^{\kappa-1} y_{2}(x, \lambda) \in L^{p}$ in the neighbourhood of zero.

A similar result is true for the bounded $V$.

Lemma 4.14. Assume that $\kappa \in \mathbb{Z} \backslash 0, \lambda \in \mathbb{C}$, and $V \in L_{2 \times 2}^{\infty}$, that is, $V$ is bounded in the neighbourhood of zero. Then, the entries of the bounded in the neighbourhood of zero solution y of (4.2) have the property:

If $\kappa>0$, then $x^{-\kappa-1} y_{1}(x, \lambda) \in L^{\infty}$ in the neighbourhood of zero, and if $\kappa<0$, then $x^{\kappa-1} y_{2}(x, \lambda) \in L^{\infty}$ in the neighbourhood of zero.

Proof. Lemma 4.14 easily follows from the proof of Lemma A1 [4]. Consider, for instance, the case $\kappa>0$. When $V=0$, equation (4.2) has a solution $\phi_{0}=\operatorname{col}\left[\begin{array}{ll}\phi_{1}^{0} & \phi_{2}^{0}\end{array}\right]$, such that the functions $x^{-\kappa-1} \phi_{1}^{0}(x, \lambda)$ and $x^{-\kappa} \phi_{2}^{0}(x, \lambda)$ are bounded in the neighbourhood of zero, and (4.2) has another

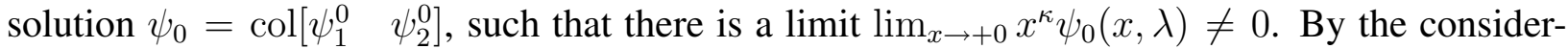
ations of [4], the bounded solution $y$ of the Dirac equation (4.2) with a bounded $V$ satisfies the integral equation

$$
\begin{gathered}
(I-\mathcal{L}) y=\gamma, \quad \mathcal{L}:=\psi_{0}(x, \lambda) \int_{0}^{x}\left[\phi_{1}^{0}(t, \lambda) \quad \phi_{2}^{0}(t, \lambda)\right] V(t) \cdot d t, \\
\gamma(x, \lambda):=\phi_{0}(x, \lambda)\left(1-\int_{0}^{x}\left[\psi_{1}^{0}(t, \lambda) \quad \psi_{2}^{0}(t, \lambda)\right] V(t) y(t, \lambda) d t\right), \quad 0<x<\varepsilon .
\end{gathered}
$$


It follows from the properties of $\phi_{0}$, Lemma 4.13, and definition (4.75) that

$$
x^{-\kappa-1} \gamma_{1}(x, \lambda) \in L^{\infty}, \quad x^{-\kappa} \gamma_{2}(x, \lambda) \in L^{\infty} \quad\left(\gamma=\operatorname{col}\left[\begin{array}{ll}
\gamma_{1} & \gamma_{2}
\end{array}\right], 0<x<\varepsilon\right) .
$$

Using (4.74) and (4.76), by induction we get for some $C>0$ and $\varepsilon(C)>0$ that

$$
\left\|\left(\mathcal{L}^{j} \gamma\right)(x)\right\| \leq C^{j+1} x^{j+\kappa}, \quad j>0, \quad 0<x<\varepsilon,
$$

where $\|\cdot\|$ is the usual vector norm. As $y=\sum_{j=0}^{\infty} \mathcal{L}^{j} \gamma$, it follows from (4.76) and (4.77) that $x^{-\kappa-1} y_{1}(x, \lambda) \in L^{\infty}$. For the case $\kappa<0$ the proof is similar.

Notice that from $S(x)=\int_{0}^{x} \Pi(t) \Pi(t)^{*} d t>0$ one easily gets $\pi_{1}(x) \not \equiv 0$ in any neighbourhood of zero. By (4.19) and by the third equality in (4.18) we see that $\breve{J}^{*} \pi_{1}^{*}$ satisfies (4.2), where $\lambda=A_{11}^{*}$. Therefore, the next corollary immediately follows from Lemmas 4.13 and 4.14.

Corollary 4.15. Let the conditions of Lemma 4.12 be fulfilled, where $1 \leq p \leq \infty$. Then for the entries $h_{1}$ and $h_{2}$ of $\pi_{1}=\left[\begin{array}{ll}h_{1} & h_{2}\end{array}\right]$, for some $\varepsilon>0$ and for some $c \in \mathbb{C} \backslash 0$ we have

$$
\begin{aligned}
& \lim _{x \rightarrow+0} x^{-\kappa} h_{1}(x)=c \neq 0, \quad x^{-\kappa-1} h_{2}(x) \in L^{p}(0, \varepsilon) \quad \text { if } \quad \kappa>0, \\
& \lim _{x \rightarrow+0} x^{\kappa} h_{2}(x)=c \neq 0, \quad x^{\kappa-1} h_{1}(x) \in L^{p}(0, \varepsilon), \quad \text { if } \quad \kappa<0 .
\end{aligned}
$$

Proof of Lemma 4.12. In a similar to the proof of Lemma 4.11 way one shows that the conditions of Proposition 4.7 are fulfilled and so (4.48) is true.

From $S(x)>0$ it follows that $\left(T_{22}^{-1}\right)(x)>0$ for $x>0$, that is, the second relation in (4.69) is valid. Taking into account the equality $S(0)=0$, formula (4.47) and Corollary 4.15 we see that

$$
S_{11}(x)=\int_{0}^{x} \pi_{1}(t) \pi_{1}(t)^{*} d t=(2|k|+1)^{-1}|c|^{2} x^{2|k|+1}+o\left(x^{2|k|+1}\right), \quad x \rightarrow+0 .
$$

Moreover, in view of (4.61) and Corollary 4.15 we obtain

$$
S_{21}(x)=\int_{0}^{x} P_{2} \Pi(t) \pi_{1}(t)^{*} d t=(|k|+1)^{-1} x^{|k|+1} P_{2} \Pi(0) \pi_{1}(0)^{*}+o\left(x^{|k|+1}\right) .
$$

Using the second relation in (4.40) and formulae (4.80) and (4.81), we get the first relation in (4.69). From the definition of $\pi_{1}$ in (4.45) and formula (4.71) it follows:

$$
\pi_{2}=-S_{21} S_{11}^{-1} \pi_{1}+P_{2} \Pi .
$$

By (4.78)-(4.82) the matrix function $\pi_{2}$ is continuous at zero. Hence, the third relation in (4.69) follows from the first relations in (4.46) and (4.69).

It remains to prove (4.73). For this purpose we shall follow a nice scheme from [4]. According to (4.78) and (4.80) we have

$$
\breve{J} \pi_{1}^{*} S_{11}^{-1} \pi_{1} \breve{J}^{*}-\pi_{1}^{*} S_{11}^{-1} \pi_{1}=-\frac{h_{1} \bar{h}_{1}}{S_{11}} \sigma_{3}+\Upsilon_{1}=-\frac{\pi_{1} \pi_{1}^{*}}{S_{11}} \sigma_{3}+\Upsilon_{2} \quad \text { for } \kappa>0,
$$


where $\Upsilon_{1}, \Upsilon_{2} \in L_{2 \times 2}^{p}$. (In the proof of this lemma functions $\Upsilon_{j}(1 \leq j \leq 8)$ are considered in the neighbourhood of zero.) According to (4.79) and (4.80) we have

$$
\breve{J} \pi_{1}^{*} S_{11}^{-1} \pi_{1} \breve{J}^{*}-\pi_{1}^{*} S_{11}^{-1} \pi_{1}=\frac{h_{2} \bar{h}_{2}}{S_{11}} \sigma_{3}+\Upsilon_{3}=\frac{\pi_{1} \pi_{1}^{*}}{S_{11}} \sigma_{3}+\Upsilon_{4} \quad \text { for } \kappa<0
$$

where $\Upsilon_{j} \in L_{2 \times 2}^{p}(j=3,4)$. In view of (4.47), rewrite $\pi_{1} \pi_{1}^{*} S_{11}^{-1}$ in the form

$$
\frac{\pi_{1}(x) \pi_{1}(x)^{*}}{S_{11}(x)}=\frac{d}{d x} \ln S_{11}(x)=\frac{2|\kappa|+1}{x}+\frac{g_{x}(x)}{g(x)}, \quad g(x):=x^{-2|\kappa|-1} S_{11}(x) .
$$

From (4.49), (4.72) and (4.83)-(4.85) it follows that

$$
\begin{gathered}
\widehat{q}_{0}(x)=-\frac{1+\kappa}{x} \sigma_{3}-\frac{g_{x}(x)}{g(x)} \sigma_{3}+\Upsilon_{5}(x) \text { for } \quad \kappa>0, \\
\widehat{q}_{0}(x)=\frac{1-\kappa}{x} \sigma_{3}+\frac{g_{x}(x)}{g(x)} \sigma_{3}+\Upsilon_{6}(x) \text { for } \quad \kappa<0,
\end{gathered}
$$

where $\Upsilon_{j} \in L_{2 \times 2}^{p}(j=5,6)$. Put

$$
f(x):=x^{-2|k|} \pi_{1}(x) \pi_{1}(x)^{*}=x^{-2|k|} \pi_{1}(x) \breve{J} \breve{J}^{*} \pi_{1}(x)^{*} .
$$

Recall that $\breve{J} \pi^{*}$ satisfies Dirac equation (4.2) with $q_{0}$ of the form (4.72), where the entries of $V$ belong $L^{p}$. Hence, differentiating (4.88) and taking into account Corollary 4.15 we get

$$
\frac{d}{d x} f(x):=-\frac{2|\kappa|}{x^{2|k|+1}} \pi_{1}(x) \pi_{1}(x)^{*}+x^{-2|k|} \pi_{1}(x) \breve{J}\left(-\frac{2 \kappa}{x} \sigma_{3}\right) \breve{J}^{*} \pi_{1}(x)^{*}+\Upsilon_{7}(x),
$$

where $\Upsilon_{7} \in L^{p}$. Apply again Corollary 4.15 to the right-hand side of (4.89) to obtain

$$
\frac{d}{d x} f(x)=-\frac{2|\kappa|}{x^{2|k|+1}} \pi_{1}(x) \pi_{1}(x)^{*}+\frac{2 \kappa}{x^{2|k|+1}} \pi_{1}(x) \sigma_{3} \pi_{1}(x)^{*}+\Upsilon_{7}(x)=\Upsilon_{8}(x),
$$

where $\Upsilon_{8} \in L^{p}$. Next, use (4.80), (4.88) and (4.90) to rewrite $S_{11}$ in the form

$$
S_{11}(x)=\int_{0}^{x} t^{2|\kappa|} f(t) d t=\frac{x^{2|k|+1}}{2|k|+1} f(x)-\frac{1}{2|k|+1} \int_{0}^{x} t^{2|\kappa|+1} \frac{d f}{d t}(t) d t .
$$

Taking into account (4.85) and (4.91), we have

$$
g(x)=\frac{1}{2|k|+1}\left(f(x)-x^{-2|k|-1} \int_{0}^{x} t^{2|\kappa|+1} \frac{d f}{d t}(t) d t\right),
$$

which implies

$$
\frac{d}{d x} g(x)=\left(\mathcal{K} f_{x}\right)(x), \quad \mathcal{K}:=x^{-2|k|-2} \int_{0}^{x} t^{2|\kappa|+1} \cdot d t
$$


As $\mathcal{K}$ is a bounded in $L^{p}(0, \varepsilon)(1 \leq p \leq \infty)$ Hardy operator, formulae (4.90) and (4.92) imply

$$
\frac{d g}{d x}(x) \in L^{p}(0, \varepsilon) .
$$

From (4.86), (4.87), and (4.93) follow relations (4.73), which completes the proof.

Proof of Theorem 4.9. Using (4.57) and (4.58) one can see that (4.22) holds at $x_{0}=0$. As $q_{0}$ is bounded, the condition of Remark 4.2 is satisfied and identity (4.22) at $x_{0}=0$ implies (4.23). Hence, the conditions of Proposition 4.7 are fulfilled. Therefore, the GBDT generated by the parameter matrices $A, S(0)$, and $\Pi(0)$ is a superposition of two GBDTs, where the first GBDT is generated by $\mathcal{A}_{1}, \mathcal{S}_{1}$, and $\Psi_{1}(0)$ (in addition to Proposition 4.7 see also (4.41)-(4.43) and Remark 4.6). As $\mathcal{S}_{1}>0$ and $q_{0}$ is bounded in the neighborhood of zero, so the transformation of $q_{0}$, which is generated by $\mathcal{A}_{1}, \mathcal{S}_{1}, \Psi_{1}(0)$, is bounded too. Denote this transformation by $q_{0}^{(1)}$.

The second GBDT in the superposition is determined by the $\varkappa \times \varkappa$ matrix $\mathcal{A}_{2}$ and by the matrix functions $T_{22}^{-1}(x)$, and $\pi_{2}(x)$. As $S_{21}(0)=0$ and $\mathcal{S}_{1}>0$ formula (4.71) implies $\lim _{x \rightarrow+0} \pi_{2}(x)=$ $\Psi_{2}(0)$. According to the second relation in (4.40) we have $\lim _{x \rightarrow+0} T_{22}^{-1}(x)=0$, that is, we may put $T_{22}^{-1}(0)=0$. Thus, the second GBDT is generated by the parameter matrices $\mathcal{A}_{2}, T_{22}^{-1}(0)=0$, and $\pi_{2}(0)=\Psi_{2}(0)$, which satisfy the identity $\mathcal{A}_{2} T_{22}^{-1}(0)-T_{22}^{-1}(0) \mathcal{A}_{2}^{*}=\Psi_{2}(0) \breve{J} \Psi_{2}(0)^{*}$.

If $\varkappa=1$, then the potential $q_{0}^{(1)}$ and the $S$-node $\mathcal{A}_{2}, T_{22}^{-1}(0)=0$, and $\pi_{2}(0)=\Psi_{2}(0)$ satisfy the conditions of Lemma 4.10 and the statement of the theorem is true.

If $\varkappa>1$, then $\mathcal{A}_{2}, T_{22}^{-1}(0)=0$ and $\pi_{2}(0)$ satisfy the conditions of Lemma 4.11 , and the second GBDT is itself a superposition of GBDTs. Taking into account the block representation

$$
\mathcal{A}_{2}=\left[\begin{array}{cc}
\mathcal{A}^{(1)} & 0 \\
* & \mathcal{A}_{2}^{(2)}
\end{array}\right], \quad \mathcal{A}^{(1)} \in \mathbb{C} ; \quad \pi_{2}=\left[\begin{array}{c}
\pi^{(1)} \\
*
\end{array}\right], \quad \pi^{(1)} \in \mathbb{C}^{2}
$$

we derive that the transformation of $q_{0}^{(1)}$ generated by $\mathcal{A}_{2}, 0$, and $\pi_{2}(0)$ is a superposition of the transformation generated by $\mathcal{A}^{(1)}, 0$, and $\pi^{(1)}(0)$, and of the transformation generated by $\mathcal{A}_{2}^{(2)}$, 0 , and $\pi_{2}^{(2)}(0)$, respectively, where $\pi_{2}^{(2)}$ is constructed analogously to the construction of $\pi_{2}$ in Proposition 4.7. (The transformation of $q_{0}^{(1)}$ generated by $\mathcal{A}^{(1)}, 0$, and $\pi^{(1)}(0)$ is denoted by $q_{0}^{(2)}$.) Moreover, for $\varkappa>2$ the potential $q_{0}^{(2)}$ and the $S$-node $\mathcal{A}_{2}^{(2)}, 0$, and $\pi_{2}^{(2)}(0)$ satisfy the conditions of Lemma 4.12, and according to Lemma 4.12 this holds for further $\varkappa-3$ steps too. At each step we obtain a new coefficient $q_{0}^{(s)}$. The absolute value of $\kappa^{(s)}$ in the representation (4.72) of $q_{0}^{(s)}(s>1)$ equals $s-1$, and the sign of $\kappa$ changes at each step till we come to the final transformation.

If $\varkappa>1$, the final transformation is generated by

$$
\mathcal{A}_{2}^{(\varkappa)}=\left(\mathcal{A}_{2}\right)_{\varkappa \varkappa} \in \mathbb{C}, \quad 0, \quad \pi_{2}^{(\varkappa)}(0)=(0,0) .
$$

Though we do not apply Lemma 4.12 at this last step directly, the proof of the representation of $\widehat{q}_{0}$ from Lemma 4.12 remains valid for $\widetilde{q}_{0}=q_{0}^{(\varkappa+1)}$, which completes the proof. 


\section{Direct and inverse problems}

In this section we consider transformed systems constructed in Section 2. assuming that the initial systems are trivial, that is, either $v \equiv 0$ or $\xi \equiv 0$. We use the notion of a minimal relization and some other notions from system theory, which are defined in Appendix A.

\subsection{Dirac systems}

\subsubsection{Self-adjoint Dirac system}

First, consider the self-adjoint Dirac system constructed in Proposition 2.1

$$
\frac{d}{d x} \widetilde{u}(x, \lambda)=i(\lambda j+j \widetilde{V}(x)) \widetilde{u}(x, \lambda) \quad(0 \leq x<\infty), \quad \widetilde{V}=\left[\begin{array}{cc}
0 & \widetilde{v} \\
\widetilde{v}^{*} & 0
\end{array}\right],
$$

$\widetilde{u}(0, \lambda)=I_{m}$. We assume that

$$
S(0)=I_{n}>0, \quad A-A^{*}=i \Pi(0) j \Pi(0)^{*}, \quad v(x) \equiv 0,
$$

where $v$ is the potential of the initial Dirac system (2.1). Partition $\Pi$ into two $n \times p$ blocks. Using (2.20), where $V \equiv 0$, we derive

$$
\Pi(x)=\left[\Phi_{1}(x) \quad \Phi_{2}(x)\right]=\left[e^{-i x A} \Phi_{1}(0) \quad e^{i x A} \Phi_{2}(0)\right] .
$$

It follows from (2.7), (2.26), and (5.2) that

$$
\begin{aligned}
& \widetilde{v}(x)=-2 i \Phi_{1}(0)^{*} e^{i x A^{*}} S(x)^{-1} e^{i x A} \Phi_{2}(0), \\
& S(x)=I_{n}+\int_{0}^{x} \Pi(t) \Pi(t)^{*} d t>0 .
\end{aligned}
$$

As $S(0)=I_{n}$ is fixed, parameter matrices in (5.3)-(5.5) are $A$ and $\Pi(0)$ or, equivalently, $A, \Phi_{1}(0)$, and $\Phi_{2}(0)$.

Definition 5.1. [28] The potentials $\widetilde{v}$ of the form (5.4) are called pseudo-exponential and the class of such potentials is denoted by PE.

If $\widetilde{v}$ is locally summable on $[0, \infty)$ (not necessarily $\widetilde{v} \in \mathrm{PE}$ ), there is a unique Weyl function of system (5.1) which is defined in the following way.

Definition 5.2. A holomorphic $p \times p$ matrix function $\varphi$ such that

$$
\begin{aligned}
& \int_{0}^{\infty}\left[\begin{array}{ll}
I_{p} & i \varphi(\lambda)^{*}
\end{array}\right] K \widetilde{u}(x, \lambda)^{*} \widetilde{u}(x, \lambda) K^{*}\left[\begin{array}{c}
I_{p} \\
-i \varphi(\lambda)
\end{array}\right] d x<\infty, \\
& \lambda \in \mathbb{C}_{+}, \quad \widetilde{u}(0, \lambda)=I_{m}, \quad K:=\frac{1}{\sqrt{2}}\left[\begin{array}{cc}
I_{p} & -I_{p} \\
I_{p} & I_{p}
\end{array}\right]
\end{aligned}
$$

is called a Weyl function of Dirac-type system (5.1) on $[0, \infty)$. 
Moreover, the function $\varphi$, which satisfies (5.6), is unique even without the analyticity requirement (see, for instance, [62]).

Now, we shall again consider the case $\widetilde{v} \in \mathrm{PE}$. As $V=0$, we have $u(x, \lambda)=\exp (i x \lambda j)$ in (2.18), and so formula (2.18) takes the form

$$
\widetilde{u}(x, \lambda)=w_{A}(x, \lambda) e^{i x \lambda j} w_{A}(0, \lambda)^{-1} .
$$

By Proposition 6.2 [33] for every $\lambda \in \mathbb{C}$, excluding a finite set of points, there is a limit

$$
\lim _{x \rightarrow \infty} w_{A}(x, \lambda)=f_{A}(\lambda) .
$$

If the equality

$$
K^{*}\left[\begin{array}{c}
I_{p} \\
-i \varphi(\lambda)
\end{array}\right]=w_{A}(0, \lambda)\left[\begin{array}{c}
I_{p} \\
0
\end{array}\right] c(\lambda)
$$

holds for some $p \times p$ matrices $c(\lambda)$ and $\varphi(\lambda)\left(\lambda \in \mathbb{C}_{+}\right)$, then formulas (5.8) and (5.9), and exponential decay of $e^{i x \lambda}$ imply inequality (5.6). As $K^{*}=K^{-1}$, it is easy to see that (5.10) is everywhere, excluding a finite set, equivalent to the relation

$$
\varphi(\lambda)=i\left(\left[\begin{array}{ll}
0 & I_{p}
\end{array}\right] K w_{A}(0, \lambda)\left[\begin{array}{c}
I_{p} \\
0
\end{array}\right]\right)\left(\left[\begin{array}{ll}
I_{p} & 0
\end{array}\right] K w_{A}(0, \lambda)\left[\begin{array}{c}
I_{p} \\
0
\end{array}\right]\right)^{-1} .
$$

Taking into account definitions (2.11) and (5.7) of $w_{A}$ and $K$, respectively, and formula (A4), one gets the following result (see [28] and Theorem 5.1 in [33]).

Theorem 5.3. Let $\widetilde{v} \in \mathrm{PE}$. Then the Weyl function of the Dirac system (5.1) admits realization

$$
\varphi(\lambda)=i I_{p}+2 \Phi_{2}(0)^{*}\left(\lambda I_{n}-\breve{A}\right)^{-1} \Phi_{1}(0), \quad \breve{A}:=A-i \Phi_{1}(0)\left(\Phi_{1}(0)+\Phi_{2}(0)\right)^{*} .
$$

Note that the Weyl function $\varphi$ is a Herglotz function and admits representation

$$
\varphi(\lambda)=\nu+\int_{-\infty}^{\infty}\left(\frac{1}{t-\lambda}-\frac{t}{1+t^{2}}\right) d \tau(t), \quad\left(\nu=\nu^{*}, \tau \uparrow\right) .
$$

Here $\tau$ is the spectral function of system (5.1). See Theorem 4.3 [28], where this fact is proved and an explicit expression for $\tau$ in terms of the parameter matrices is given. The inverse problem to recover $\widetilde{v} \in \mathrm{PE}$ from $\tau$ is solved explicitly in Theorem 4.5 [28]. (The corresponding bound states are constructed in [32].) The inverse problem to recover $\widetilde{v}$ from the spectral density for the particular case $\sigma(A) \subset \mathbb{C}_{+}$was treated earlier in [5].

The left reflection coefficient for system (5.1), where $\widetilde{v} \in \mathrm{PE}$, is expressed via the Weyl function by the formula (see p. 33 in [33]):

$$
R_{L}(\lambda)=-\left(I_{p}+i \varphi(\lambda)\right)\left(I_{p}-i \varphi(\lambda)\right)^{-1}
$$

Recall that $\varphi$ has properties

$$
\Im \varphi(\lambda) \geq 0 \quad\left(\lambda \in \mathbb{C}_{+}\right), \quad \lim _{\lambda \rightarrow \infty} \varphi(\lambda)=i I_{p}
$$


It follows from (5.14) and (5.15) that

$$
\left\|R_{L}(\lambda)\right\| \leq 1 \quad\left(\lambda \in \mathbb{C}_{+}\right), \quad \lim _{\lambda \rightarrow \infty} R_{L}(\lambda)=0 .
$$

To recover system (5.1) from its Weyl function we should recover parameter matrices $A$ and $\Phi_{k}(0)(k=1,2)$. First, we recover $R_{L}$ via formula (5.14). By the second relation in $(5.16) R_{L}$ is a strictly proper matrix function and admits a minimal realization

$$
R_{L}(\lambda)=\mathcal{C}\left(\lambda I_{n}-\mathcal{A}\right)^{-1} \mathcal{B}
$$

where $\mathcal{C}$ is a $p \times n$ matrix and $\mathcal{B}$ is an $n \times p$ matrix. According to the first relation in (5.16), $R_{L}$ admitting the minimal realization (5.17) is also contractive. Hence, by Theorems 21.1.1 and 21.1.3 in [LR] the Riccati equation

$$
\mathcal{X C} \mathcal{C}^{*}-i\left(\mathcal{A X}-\mathcal{X} \mathcal{A}^{*}\right)+\mathcal{B B}^{*}=0
$$

has a positive solution $\mathcal{X}>0$. Put

$$
A=\mathcal{X}^{-\frac{1}{2}} \mathcal{A} \mathcal{X}^{\frac{1}{2}}+i \mathcal{X}^{-\frac{1}{2}} \mathcal{B} \mathcal{B}^{*} \mathcal{X}^{-\frac{1}{2}}, \quad \Phi_{1}(0)=\mathcal{X}^{-\frac{1}{2}} \mathcal{B}, \quad \Phi_{2}(0)=-i \mathcal{X}^{\frac{1}{2}} \mathcal{C}^{*}
$$

The matrix identity given by the second relation in (5.2) is immediate from (5.18) and (5.19), that is, $A$ and $\Phi_{k}(0)(k=1,2)$ satisfy the requirement on parameter matrices and we can apply Theorem 5.3. It easily follows (see Section 9 in [33]) that $\varphi$ given by (5.12) and (5.19) coincides with the $\varphi$ from which $R_{L}$ was recovered.

Theorem 5.4. [33] Let $\varphi$ be a rational $p \times p$ matrix function, which satisfies (5.15). Then $\varphi$ is the Weyl function of some system (5.1), where $\widetilde{v} \in \mathrm{PE}$. To recover $\widetilde{v}$ we take a minimal realization (5.17) of $R_{L}$ given by (5.14) and define parameter matrices by (5.19), where $\mathcal{X}>0$ is a solution of the Riccati equation (5.18). After that we apply formulas (5.3)-(5.5).

By Theorem 5.4 [33] all the potentials $\widetilde{v} \in \mathrm{PE}$ admit representation (5.4), where parameter matrices have additional properties

$$
\sigma(A) \subset \overline{\mathbb{C}}_{+}, \quad \operatorname{span} \bigcup_{k=0}^{n-1} \operatorname{Im} A^{k} \Phi_{1}(0)=\mathbb{C}^{n}, \quad \operatorname{span} \bigcup_{k=0}^{n-1} \operatorname{Im} A^{k} \Phi_{2}(0)=\mathbb{C}^{n}
$$

and Im means image. Hence, without loss of generality we can assume (5.20).

\subsubsection{Potentials with singularities}

Now, consider a more general case of Dirac systems, including systems with singularities. Namely, let

$$
j=\left[\begin{array}{cc}
I_{p_{1}} & 0 \\
0 & -I_{p_{2}}
\end{array}\right], \quad S(0)=S_{0}, \quad A S_{0}-S_{0} A^{*}=i \Pi(0) j \Pi(0)^{*},
$$


where $p_{1}, p_{2}>0, p_{1}+p_{2}=m$. Formula (5.5) takes the form

$$
S(x)=S_{0}+\int_{0}^{x} \Pi(t) \Pi(t)^{*} d t .
$$

As before, $\Pi, w_{A}$, and $\widetilde{v}$ are defined via formulas (5.3), (2.11), and (5.4), respectively, and we assume that (5.20) holds. Here $\Phi_{1}, \Phi_{2}$, and $\widetilde{v}$ are $n \times p_{1}, n \times p_{2}$, and $p_{1} \times p_{2}$ matrix functions, respectively.

Definition 5.5. [22] The potentials $\widetilde{v}$ of the form (5.4), where (5.20) and (5.21) hold, and $S$ is given by (5.22), are called generalized pseudo-exponential and the class of such potentials is denoted by GPE.

Dirac system with $\widetilde{v} \in$ GPE was treated on the whole axis in [63], and results on supertransparent potentials and soliton-positon interactions were obtained. In particular, it was shown in the proof of Theorem 2.1 from [63] that there are values $x_{0} \in \mathbb{R}$ such that

$$
S(x)>0 \text { for all } x>x_{0} .
$$

If $S_{0} \ngtr 0$, then det $S\left(x_{k}\right)=0$ in some point (or points) on $\mathbb{R}_{+} \cup\{0\}$, which means that the GPE class includes potentials with singularities. Nevertheless, as $S(x)$ admits continuation meromorphic in $x$, the determinant det $S(x)$ turns into zero only in a finite number of points on $\mathbb{R}_{+} \cup\{0\}$. The proof that the matrix function

$$
\widetilde{u}(x, \lambda)=w_{A}(x, \lambda) e^{i x \lambda j}
$$

satisfies (5.1) and is nondegenerate (excluding a finite number of values of $\lambda$ and zeros of det $S(x)$ ) remains true for Dirac system with $j$ given by (5.21). Moreover, $\widetilde{u}$ as well as $S$ is meromorphic in $x$. Therefore, we call $\widetilde{u}$ a fundamental solution of (5.1), which agrees with the standard [46] requirement for the fundamental and special solutions in the case of singularities to be defined by the same formula on the whole domain.

The asymptotics of $w_{A}$ in (5.24) under condition (5.20) is given in the next proposition (see Proposition 3.1 in [22] or Theorem 3.1 in [63]).

Proposition 5.6. Let $A, S(0)$, and $\Phi_{k}(0)(k=1,2)$ satisfy (5.20) and (5.21). Then there is a limit

$$
\omega=\lim _{x \rightarrow \infty}\left(e^{-i x A} S(x) e^{i x A^{*}}\right)^{-1} \geq 0
$$

and we have

$$
\lim _{x \rightarrow \infty} w_{A}(x, \lambda)=\left[\begin{array}{lr}
I_{p_{1}} & 0 \\
0 & \chi(\lambda)
\end{array}\right], \quad \chi(\lambda):=I_{p_{2}}+i \Phi_{2}(0)^{*} \omega\left(A-\lambda I_{n}\right)^{-1} \Phi_{2}(0) .
$$


Transmission and reflection coefficients for system (5.1) are defined [22, 33, 82] in terms of special solutions of (5.1), that is, $m \times p_{1}$ and $m \times p_{2}$ solutions $\mathcal{Y}$ and $\mathcal{Z}$, respectively, which we determine by the relations

$$
\mathcal{Y}(x, \lambda)=e^{i x \lambda}\left[\begin{array}{c}
I_{p_{1}} \\
0
\end{array}\right]+o(1) \quad(x \rightarrow \infty), \quad \mathcal{Z}(0, \lambda)=\left[\begin{array}{c}
0 \\
I_{p_{2}}
\end{array}\right], \quad \lambda \in \mathbb{R} .
$$

Put

$$
\begin{aligned}
& T_{L}(\lambda):=\mathcal{Y}_{1}(0, \lambda)^{-1}, \quad R_{L}(\lambda):=\mathcal{Y}_{2}(0, \lambda) \mathcal{Y}_{1}(0, \lambda)^{-1}, \\
& R_{R}(\lambda):=\left(\lim _{x \rightarrow \infty} e^{-i x \lambda} \mathcal{Z}_{1}(x, \lambda)\right)\left(\lim _{x \rightarrow \infty} e^{i x \lambda} \mathcal{Z}_{2}(x, \lambda)\right)^{-1}, \\
& T_{R}(\lambda):=\left(\lim _{x \rightarrow \infty} e^{i x \lambda} \mathcal{Z}_{2}(x, \lambda)\right)^{-1} .
\end{aligned}
$$

Here the $p_{1} \times p_{1}$ matrix $\mathcal{Y}_{1}$ and the $p_{1} \times p_{2}$ matrix $\mathcal{Y}_{2}$ are upper and lower blocks of $\mathcal{Y}$, respectively. Analogously, the $p_{1} \times p_{2}$ matrix $\mathcal{Z}_{1}$ and the $p_{2} \times p_{2}$ matrix $\mathcal{Z}_{2}$ are upper and lower blocks of $\mathcal{Z}$, respectively. The functions $T_{L}$ and $T_{R}$ are called the left and the right transmission coefficients and $R_{L}$ and $R_{R}$ are called the left and the right reflection coefficients, respectively.

Using (5.24) and (5.26) we get the result

Theorem 5.7. [22] Let $\widetilde{v} \in$ GPE. Then the transmission and reflection coefficients are given by the formulas

$$
\begin{aligned}
T_{L}(\lambda)= & I_{p_{1}}+i \Phi_{1}(0)^{*} S_{0}^{-1}\left(\theta-\lambda I_{n}\right)^{-1} \Phi_{1}(0) \\
R_{L}(\lambda)= & i \Phi_{2}(0)^{*} S_{0}^{-1}\left(\theta-\lambda I_{n}\right)^{-1} \Phi_{1}(0) \\
T_{R}(\lambda)= & I_{p_{2}}+i \Phi_{2}(0)^{*} S_{0}^{-1}\left(\theta-\lambda I_{n}\right)^{-1}\left(I_{n}-S_{0} \omega\right) \Phi_{2}(0) \\
R_{R}(\lambda)= & i \Phi_{1}(0)^{*} S_{0}^{-1}\left(\theta-\lambda I_{n}\right)^{-1}\left(I_{n}-S_{0} \omega\right) \Phi_{2}(0) \\
& +i \Phi_{1}(0)^{*}\left(A^{*}-\lambda I_{n}\right)^{-1} \omega \Phi_{2}(0)
\end{aligned}
$$

where $\theta:=A-i \Phi_{1}(0) \Phi_{1}(0)^{*} S_{0}^{-1}$.

The inverse problem to recover $\widetilde{v}$ from $R_{L}$ is solved in Theorem 4.1 [22].

Theorem 5.8. Let $\mathcal{R}$ be a strictly proper rational $p_{2} \times p_{1}$ matrix function. Then $\mathcal{R}$ is the left reflection coefficient of a system (5.1) with $v \in \mathrm{GPE}$ if and only if $\mathcal{R}$ is contractive on $\mathbb{R}$. If $\mathcal{R}$ satisfies this condition, then $\widetilde{v}$ can be uniquely recovered from $\mathcal{R}$ in two steps, that is, steps $(i)$ and (ii) below.

(i) First, take a minimal realization

$$
\mathcal{R}(\lambda)=\mathcal{C}\left(\lambda I_{n}-\mathcal{A}\right)^{-1} \mathcal{B}
$$

Then there is a unique solution $\mathcal{X}$ of the Riccati equation

$$
i\left(\mathcal{X} \mathcal{A}-\mathcal{A}^{*} \mathcal{X}\right)=\mathcal{C}^{*} \mathcal{C}+\mathcal{X B B}{ }^{*} \mathcal{X}
$$


such that

$$
\sigma\left(\mathcal{A}+i \mathcal{B B} \mathcal{B}^{*} \mathcal{X}\right) \subset \overline{\mathbb{C}}_{-}, \quad \mathcal{X}=\mathcal{X}^{*}
$$

Moreover, $\operatorname{det} \mathcal{X} \neq 0$.

(ii) Next, recover parameter matrices by the equalities

$$
A=\mathcal{A}+i \mathcal{B B}^{*} \mathcal{X}, \quad S_{0}=\mathcal{X}^{-1}, \quad \Phi_{1}(0)=\mathcal{B}, \quad \Phi_{2}(0)=-i S_{0} \mathcal{C}^{*},
$$

and recover $\widetilde{v}$ via (5.3), (5.4), and (5.22).

\subsubsection{Skew-self-adjoint Dirac system}

In this subsubsection we consider system

$$
\begin{gathered}
\frac{d}{d x} \widetilde{u}(x, \lambda)=(i \lambda j+j \widetilde{V}(x)) \widetilde{u}(x, \lambda) \quad(0 \leq x<\infty), \quad \widetilde{V}=\left[\begin{array}{cc}
0 & \widetilde{v} \\
\widetilde{v}^{*} & 0
\end{array}\right], \\
\widetilde{v}(x)=2 \Phi_{1}(0)^{*} e^{i x A^{*}} S(x)^{-1} e^{i x A} \Phi_{2}(0), \quad S(x)=I_{n}+\int_{0}^{x} \Pi(t) j \Pi(t)^{*} d t,
\end{gathered}
$$

where $j$ has the form (1.9), $\Pi$ is given by (5.3), and the identity $A-A^{*}=i \Pi(0) \Pi(0)^{*}$ is fulfilled. That is, we consider GBDT of the trivial initial system, where $v \equiv 0$. According to (2.23) and to the second relation in (5.40) the inequalities $\frac{d}{d x}\left(e^{-i x A} S(x) e^{-i x A^{*}}\right) \geq 0$ and $\left.\left(e^{-i x A} S(x) e^{-i x A^{*}}\right)\right|_{x=0}>$ 0 hold, and so $S(x)>0$. Thus, the right-hand side of the first equality in (5.40) is well-defined. The class of potentials $\widetilde{v}$ of the form (5.40) is denoted by $\mathrm{PE}_{2}$. By Proposition 1.4 in [29] for each $\widetilde{v} \in \mathrm{PE}_{2}$ there is some $M>0$ such that

$$
\sup _{x \in[0, \infty)}\|\widetilde{v}(x)\| \leq M .
$$

It follows from Proposition 2.2 that the fundamental solution $\widetilde{u}(x, \lambda)$ of system (5.39) (normalized by $\left.\widetilde{u}(0, \lambda)=I_{m}\right)$ is given by formula (5.8), where $w_{A}$ has the form (2.24).

Analogously to Definition 5.2 one can define Weyl functions of the skew-self-adjoint Dirac system [16, 29, 53, 69].

Definition 5.9. Let system (5.39), where $\|\widetilde{v}(x)\|$ is bounded on all the finite intervals $[0, l]$, be given. Then, a holomorphic $p \times p$ matrix function $\varphi$ such that

$$
\int_{0}^{\infty}\left[\varphi(\lambda)^{*} I_{p}\right] \widetilde{u}(x, \lambda)^{*} \widetilde{u}(x, \lambda)\left[\begin{array}{c}
\varphi(\lambda) \\
I_{p}
\end{array}\right] d x<\infty, \quad \Im \lambda<-M_{1}
$$

is called a Weyl function of (5.39).

There is a unique Weyl function of system (5.39) with a bounded on $[0, \infty)$ potential $[53,69]$. If (5.41) holds, we can put in (5.42) $M_{1}=M$. For a generalization of the Definition 5.9 see $[16,23,54]$.

Direct and inverse problems for $\widetilde{v} \in \mathrm{PE}_{2}$ are solved explicitly in Theorems 2.1 and 2.3 from [29]. It is done in a similar to the self-adjoint case way. 
Theorem 5.10. [29] Let $v \in P E_{2}$. Then system (5.39) has a unique Weyl function $\varphi$, which satisfies (5.42) on the lower semi-plane $\mathbb{C}_{-}$, a finite number of poles excluded, and this function is given by the formula

$$
\varphi(\lambda)=i \Phi_{1}(0)^{*}\left(\lambda I_{n}-\breve{A}\right)^{-1} \Phi_{2}(0), \quad \breve{A}:=A-i \Phi_{2}(0) \Phi_{2}(0)^{*} .
$$

Theorem 5.11. [29] Let $\varphi$ be a strictly proper rational $p \times p$ matrix function. Then $\varphi$ is the Weyl function of the skew-self-adjoint Dirac system (5.42), where $\widetilde{v} \in P E_{2}$. To recover $\widetilde{v}$ take a minimal realization

$$
\varphi(\lambda)=\mathcal{C}\left(\lambda I_{n}-\mathcal{A}\right)^{-1} \mathcal{B} .
$$

There is a positive solution $\mathcal{X}>0$ of the Riccati equation

$$
\mathcal{X} \mathcal{C}^{*} \mathcal{C X}+i\left(\mathcal{A X}-\mathcal{X} \mathcal{A}^{*}\right)-\mathcal{B B}^{*}=0
$$

After putting

$$
\Phi_{1}(0)=i \mathcal{X}^{\frac{1}{2}} \mathcal{C}^{*}, \quad \Phi_{2}(0)=\mathcal{X}^{-\frac{1}{2}} \mathcal{B}, \quad A=\mathcal{X}^{-\frac{1}{2}} \mathcal{A X}^{\frac{1}{2}}+i \mathcal{X}^{-\frac{1}{2}} \mathcal{B B}^{*} \mathcal{X}^{-\frac{1}{2}},
$$

the potential $\widetilde{v}$ is recovered by formula (5.40).

\section{2. $N$-wave equation}

Here we consider GBDT of the auxiliary system for the $N$-wave equation

$$
\frac{d}{d x} \widetilde{u}(x, \lambda)=(i \lambda D-[D, \widetilde{\xi}(x)]) \widetilde{u}(x, \lambda) \quad(0 \leq x<\infty), \quad \widetilde{\xi}^{*}=\widetilde{\xi} .
$$

We consider the subcase of (2.27), where $B=I_{m}$, and assume that

$$
D=\operatorname{diag}\left\{d_{1}, d_{2}, \ldots, d_{m}\right\}, \quad d_{1}>d_{2}>\ldots>d_{m}>0 .
$$

Starting from system (2.27) with $\xi \equiv 0$ and taking into account $B=I_{m}$, rewrite $(2.31)$ as:

$$
\widetilde{\xi}=\Pi^{*} S^{-1} \Pi,
$$

where

$$
A S(0)-S(0) A^{*}=i \Pi(0) \Pi(0)^{*}, \quad \Pi_{x}=-i A \Pi D, \quad S_{x}=\Pi D \Pi^{*} .
$$

We require

$$
\widetilde{u}(0, \lambda)=I_{m}, \quad S(0)>0 .
$$

As we have $u(x, \lambda)=\exp (i x \lambda D)$ for the fundamental solution of the initial system, formula (2.18) takes the form

$$
\widetilde{u}(x, \lambda)=w_{A}(x, \lambda) e^{i x \lambda D} w_{A}(0, \lambda)^{-1},
$$

where $w_{A}$ has the form (2.24) and $A S-S A^{*}=i \Pi^{*}$. It follows from (2.24) and $A S-S A^{*}=$ $i \Pi \Pi^{*}$ that

$$
w_{A}(x, \lambda)^{*} w_{A}(x, \lambda)=I_{m}-i(\lambda-\bar{\lambda}) \Pi(x)^{*}\left(A^{*}-\lambda I_{n}\right)^{-1} S(x)^{-1}\left(A-\lambda I_{n}\right)^{-1} \Pi(x) .
$$


By the last relations in (5.50) and (5.51) we get $S(x)>0$ for $x \geq 0$, that is, $w_{A}(x, \lambda)$ is welldefined for all $x \geq 0$ and all $\lambda \notin \sigma(A)$. If $\lambda \in \mathbb{C}_{-}$, the inequality $S(x)>0$ and formula (5.53) imply

$$
\begin{aligned}
& w_{A}(x, \lambda)^{*} w_{A}(x, \lambda) \leq I_{m}, \\
& i(\lambda-\bar{\lambda}) \Pi(x)^{*}\left(A^{*}-\lambda I_{n}\right)^{-1} S(x)^{-1}\left(A-\lambda I_{n}\right)^{-1} \Pi(x) \leq I_{m} .
\end{aligned}
$$

Proposition 5.12. Let parameter matrices $A, S(0)$, and $\Pi(0)$ satisfy conditions $A S(0)-S(0) A^{*}=$ $i \Pi(0) \Pi(0)^{*}$ and $S(0)>0$. Then $\widetilde{\xi}$ determined by $(5.49)$ and $(5.50)$ is bounded on $[0, \infty)$.

Proof. It follows from (5.50) that $\Pi$ has the form

$$
\begin{aligned}
& \Pi(x)=\left[\exp \left(-i d_{1} x A\right) f_{1} \ldots \exp \left(-i d_{m} x A\right) f_{m}\right], \\
& f_{k} \in \mathbb{C}^{m} \quad(1 \leq k \leq m) .
\end{aligned}
$$

One can easily see that $\operatorname{span}_{\lambda \in \mathcal{O}}\left(A-\lambda I_{n}\right)^{-1} f_{k} \supseteq f_{k}$ for any open domain $\mathcal{O}$. Hence, by $(5.55)$ and (5.56) we have

$$
\sup _{x \in \mathbb{R}_{+}}\left\|S(x)^{-\frac{1}{2}} e^{-i d_{k} x A} f_{k}\right\|<\infty \quad(1 \leq k \leq m) .
$$

Now, the boundedness of $\widetilde{\xi}$ of the form $(5.49)$ is immediate.

The class of potentials $\widetilde{\xi}$ of the form $(5.49)$, which are generated by parameter matrices satisfying conditions of Proposition 5.12, is denoted by $\mathrm{PE}_{3}$.

Definition 5.13. [60, 74] A Weyl function of system (5.47) is an $m \times m$ matrix function $\varphi$, such that for some $M>0$ it is analytic in a lower semi-plane $\Im \lambda<-M$, and the inequalities

$$
\sup _{x \leq l, \Im \lambda<-M}\|\widetilde{u}(x, \lambda) \varphi(\lambda) \exp (-i x \lambda D)\|<\infty
$$

hold for all $l<\infty$.

In our case $\widetilde{\xi}$ is bounded, and so (see [60]) the system (5.47) has a unique Weyl function $\widetilde{\varphi}$ normalized by the condition

$$
\widetilde{\varphi}_{k s}(\lambda) \equiv 1 \text { for } k=s, \quad \widetilde{\varphi}_{k s}(\lambda) \equiv 0 \text { for } k>s .
$$

This Weyl function satisfies for some $\varepsilon>0$ the inequality

$$
\int_{0}^{\infty}(\exp (i x \bar{\lambda} D)) \widetilde{\varphi}(\lambda)^{*} \widetilde{u}(x, \lambda)^{*} \widetilde{u}(x, \lambda) \widetilde{\varphi}(\lambda) \exp \left(x\left(-i \lambda D-\varepsilon I_{m}\right)\right) d x<\infty .
$$

Here, we do not require (5.59). Our next theorem is immediate from (5.52) and (5.54). 
Theorem 5.14. A Weyl function of system (5.47), where $\widetilde{\xi} \in \mathrm{PE}_{3}$, is given by the formula

$$
\varphi(\lambda)=w_{A}(0, \lambda)=I_{m}-i \Pi(0)^{*} S(0)^{-1}\left(A-\lambda I_{n}\right)^{-1} \Pi(0) .
$$

Notice that in view of (5.53) and (5.54) the matrix function $\varphi(\lambda)$ given by (5.61) has following properties:

$$
\varphi(\lambda) \varphi(\bar{\lambda})^{*}=I_{m}, \quad \varphi(\lambda)^{*} \varphi(\lambda) \leq I_{m} \quad\left(\lambda \in \mathbb{C}_{-}\right), \quad \lim _{\lambda \rightarrow \infty} \varphi(\lambda)=I_{m}
$$

In particular, this matrix function admits a minimal realization

$$
\varphi(\lambda)=I_{m}+\mathcal{C}\left(\lambda I_{n}-\mathcal{A}\right)^{-1} \mathcal{B}
$$

It follows from the second relation in $(5.62)$ that $\sigma(\mathcal{A}) \in \mathbb{C}_{+}$. Hence, there is a unique and positive solution $S_{0}>0$ of the identity

$$
\mathcal{A} S_{0}-S_{0} \mathcal{A}^{*}=i \mathcal{B} \mathcal{B}^{*}
$$

Matrix functions admitting realization (5.62) satisfy conditions of Theorem 1.2 from [60] and there is at most one solution of the corresponding inverse problem. This solution is given in Theorem 5.6 from [74].

Theorem 5.15. [74] Let a $p \times p$ rational matrix function $\varphi$ satisfy conditions (5.62). Then $\varphi$ is a Weyl function of the unique system (5.47), where $\widetilde{\xi} \in \mathrm{PE}_{3}$. To recover $\widetilde{\xi}$ take a minimal realization (5.63) of $\varphi$, recover $S_{0}$ from (5.64), and put

$$
A=\mathcal{A}, \quad S(0)=S_{0}, \quad \Pi(0)=\mathcal{B} .
$$

Then $\widetilde{\xi}$ is generated by the parameter matrices $A, S(0)$, and $\Pi(0)$ via formulas $(5.49)$ and (5.50).

Finally, let parameter matrices $A, S(0,0)>0$, and $\Pi(0,0)$ satisfy $(2.36)$, where $B=I_{m}$, and generate via (5.49) the solution $\widetilde{\xi}$ of the $N$-wave equation (2.32). The corresponding evolution of the Weyl function is given by the formula

$$
\varphi(t, \lambda)=w_{A}(0, t, \lambda)=I_{m}-i \Pi(0, t)^{*} S(0, t)^{-1}\left(A-\lambda I_{n}\right)^{-1} \Pi(0, t) .
$$

Here $\Pi_{t}=-i A \Pi \widehat{D}, S_{t}=\Pi \widehat{D} \Pi^{*}$, and formula (5.66) holds on the interval $t \in[0, \varepsilon)$, where $S(0, t)>0$.

\section{Acknowledgement}

The work was supported by the Austrian Science Fund (FWF) under Grant no. Y330. 


\section{A Mathematical system theory}

We present here some basic results and notions from mathematical system theory of rational matrix functions that are used in our review. This material has its roots in Kalman theory [38], and can be found in various books (see, for instance, $[8,17]$ ). See also interesting historical remarks in [40].

The rational matrix functions appearing in the article are proper, that is, analytic at infinity. Such an $m_{2} \times m_{1}$ matrix function $W$ can be represented in the form

$$
W(\lambda)=\mathcal{D}+\mathcal{C}\left(\lambda I_{n}-\mathcal{A}\right)^{-1} \mathcal{B}
$$

where $\mathcal{A}$ is a square matrix of some order $n$, the matrices $\mathcal{B}$ and $\mathcal{C}$ are of sizes $n \times m_{1}$ and $m_{2} \times n$, respectively, and $\mathcal{D}=W(\infty)$. The representation (A1) is called a realization of $W$, and the number $\operatorname{ord}(\mathcal{A})$ (order of the matrix $\mathcal{A}$ ) is called the state space dimension of the realization.

The realization (A1) is said to be minimal if its state space dimension $n$ is minimal among all possible realizations of $W$. This minimal $n$ is called the McMillan degree of $W$. The realization (A1) of $W$ is minimal if and only if

$$
\operatorname{span} \bigcup_{k=0}^{n-1} \operatorname{Im} \mathcal{A}^{k} \mathcal{B}=\mathbb{C}^{n}, \quad \operatorname{span} \bigcup_{k=0}^{n-1} \operatorname{Im}\left(\mathcal{A}^{*}\right)^{k} \mathcal{C}^{*}=\mathbb{C}^{n}, \quad n=\operatorname{ord}(\mathcal{A}),
$$

where Im is image. If for a pair of matrices $\mathcal{A}, \mathcal{B}$ the first equality in (A2) holds, then the pair $\mathcal{A}$, $\mathcal{B}$ is called controllable or a full range. If the second equality in (A2) is fulfilled, then $\mathcal{C}, \mathcal{A}$ is said to be observable. If a pair $\mathcal{A}, \mathcal{B}$ is full range, and $\mathcal{K}$ is an $m_{1} \times n$ matrix, then the pair $\mathcal{A}-\mathcal{B K}, \mathcal{B}$ is also full range.

Minimal realizations are unique up to a basis transformation, that is, if (A1) is a minimal realization of $W$ and if $W(\lambda)=\mathcal{D}+\widetilde{\mathcal{C}}\left(\lambda I_{n}-\widetilde{\mathcal{A}}\right)^{-1} \widetilde{\mathcal{B}}$ is a second minimal realization of $W$, then there exists an invertible matrix $\mathcal{S}$ such that

$$
\widetilde{\mathcal{A}}=\mathcal{S} \mathcal{A S}^{-1}, \quad \widetilde{\mathcal{B}}=\mathcal{S B}, \quad \widetilde{\mathcal{C}}=\mathcal{C S}^{-1}
$$

In this case, (A3) is called a similarity transformation.

Finally, if $W$ is a square matrix and $\mathcal{D}=I_{m_{1}}\left(m_{1}=m_{2}\right)$, then $W^{-1}$ admits representation

$$
W(\lambda)^{-1}=I_{m_{1}}-\mathcal{C}\left(\lambda I_{n}-\mathcal{A}^{\times}\right)^{-1} \mathcal{B}, \quad \mathcal{A}^{\times}=\mathcal{A}-\mathcal{B C} .
$$

\section{References}

[1] M.J. Ablowitz, S. Chakravarty, A.D. Trubatch, J. Villarroel. A novel class of solutions of the non-stationary Schrödinger and the Kadomtsev-Petviashvili I equations. Phys. Lett. A, n267 (2000), No. 2-3, 132-146. 
[2] M.J. Ablowitz, R. Haberman. Resonantly coupled nonlinear evolution equations. J. Math. Phys., 16 (1975), 2301-2305.

[3] M. Adler, P. van Moerbeke. Birkhoff strata, Bäcklund transformations, and regularization of isospectral operators. Adv. Math., 108 (1994), No. 1, 140-204.

[4] S. Albeverio, R. Hryniv, Ya. Mykytyuk. Reconstruction of radial Dirac operators. J. Math. Phys. 48 (2007), No. 4, 043501, 14 pp.

[5] D. Alpay, I. Gohberg. Inverse spectral problem for differential operators with rational scattering matrix functions. J. Diff. Eqs., 118 (1995), 1-19.

[6] D. Alpay, I. Gohberg, M.A. Kaashoek, A.L. Sakhnovich. Direct and inverse scattering problem for canonical systems with a strictly pseudo-exponential potential. Math. Nachr., 215 (2000), 5-31.

[7] A.V. Bäcklund. Zur Theorie der partiellen Differential gleichungen erster Ordnung. Math. Ann., 17 (1880), 285-328.

[8] H. Bart, I. Gohberg, M.A. Kaashoek. Minimal factorization of matrix and operator functions. Operator Theory: Adv. Appl., 1, Birkhäuser Verlag, Basel, 1979.

[9] R. Beals, R.R. Coifman. Scattering and inverse scattering for first-order systems: II. Inverse Probl., 3 (1987), 577-593.

[10] A.B. Borisov, V.V. Kiseliev. Inverse problem for an elliptic sine-Gordon equation with an asymptotic behaviour of the cnoidal-wave type. Inverse Probl., 5 (1989), 959-982.

[11] A. Boutet de Monvel, V. Marchenko. Generalization of the Darboux transform. Matematicheskaya fizika, analiz, geometriya, 1 (1994), 479-504.

[12] B. Carl, C. Schiebold. Nonlinear equations in soliton physics and operator ideals. Nonlinearity, 12 (1999), 333-364.

[13] R.C. Cascaval, F. Gesztesy, H. Holden, Yu. Latushkin. Spectral analysis of Darboux transformations for the focusing NLS hierarchy. J. Anal. Math., 93 (2004), 139-197.

[14] D.V. Chudnovsky, G.V. Chudnovsky. Bäcklund transformation as a method of decomposition and reproduction of two-dimensional nonlinear systems. Phys. Lett. A, 87 (1982), No. 7 , $325-329$.

[15] J. Cieslinski. An effective method to compute $N$-fold Darboux matrix and $N$-soliton surfaces. J. Math. Phys., 32 (1991), 2395-2399.

[16] S. Clark, F. Gesztesy. On self-adjoint and J-self-adjoint Dirac-type operators: a case study. Contemporary Mathematics, 412 (2006), 103-140. 
[17] M.J. Corless, A.E. Frazho. Linear Systems and Control - An Operator Perspective. Marcel Dekker, New York, 2003.

[18] M.M. Crum. Associated Sturm-Liouville systems. Quart. J. Math. Oxford Ser. (2), 6 (1955), $121-127$.

[19] G. Darboux. Lecons sur la Theorie Generale de Surface et les Applications Geometriques du Calcul Infinitesimal, II. Gauthiers-Villars, Paris, 1889.

[20] P.A. Deift. Applications of a commutation formula. Duke Math. J., 45 (1978), 267-310.

[21] L.D. Faddeev, L.A. Takhtajan. Hamiltonian methods in the theory of solitons. Springer Verlag, NY, 1986.

[22] B. Fritzsche, B. Kirstein, A.L. Sakhnovich. Completion problems and scattering problems for Dirac type differential equations with singularities. J. Math. Anal. Appl., 317 (2006), $510-525$.

[23] B. Fritzsche, B. Kirstein, A.L. Sakhnovich. Semiseparable integral operators and explicit solution of an inverse problem for the skew-self-adjoint Dirac-type system. arXiv:0904.2357

[24] F. Gesztesy. A complete spectral characterization of the double commutation method. J. Funct. Anal., 117 (1993), No. 2, 401-446.

[25] F. Gesztesy, H. Holden. Soliton equations and their algebro-geometric solutions. Cambridge Studies in Advanced Mathematics, 79, Cambridge University Press, Cambridge, 2003.

[26] F. Gesztesy, B. Simon, G. Teschl. Spectral deformations of one-dimensional Schrödinger operators. J. Anal. Math., 70 (1996), 267-324.

[27] F. Gesztesy, G. Teschl. On the double commutation method. Proc. Am. Math. Soc., 124 (1996), No. 6, 1831-1840.

[28] I. Gohberg, M.A. Kaashoek, A.L. Sakhnovich. Canonical systems with rational spectral densities: explicit formulas and applications. Mathematische Nachr. 194 (1998), 93-125.

[29] I. Gohberg, M.A. Kaashoek, A.L. Sakhnovich. Pseudocanonical systems with rational Weyl functions: explicit formulas and applications. J. Differ. Equations, 146 (1998), 375-398.

[30] I. Gohberg, M.A. Kaashoek, A.L. Sakhnovich. Sturm-Liouville systems with rational Weyl functions: explicit formulas and applications. IEOT, 30 (1998), 338-377.

[31] I. Gohberg, M.A. Kaashoek, A.L. Sakhnovich. Canonical systems on the full line with rational spectral densities: explicit formulas. In: Operator Theory: Adv. Appl., 117, M.G. Krein volume (2000), 127-139.

[32] I. Gohberg, M.A. Kaashoek, A.L. Sakhnovich. Bound states for canonical systems on the half and full line: explicit formulas. IEOT, 40 (2001), No. 3, 268-277. 
[33] I. Gohberg, M.A. Kaashoek, A.L. Sakhnovich. Scattering problems for a canonical system with a pseudo-exponential potential. Asymptotic Analysis, 29 (2002), No. 1, 1-38.

[34] C.H. Gu, H. Hu, Z. Zhou. Darboux transformations in integrable systems. Springer Verlag, 2005.

[35] C.G.T. Jacobi. Über eine neue Methode zur Integration der hyperelliptischen Differentialgleichungen und über die rationale Form ihrer vollständigen algebraischen Integralgleichungen. J. Reine Angew. Math., 32 (1846), 220-226.

[36] M. Jaworski, D. Kaup. Direct and inverse scattering problem associated with the elliptic sinh-Gordon equation. Inverse Problems, 6 (1990), 543-556.

[37] M.A. Kaashoek, A.L. Sakhnovich. Discrete skew self-adjoint canonical system and the isotropic Heisenberg magnet model. J. Funct. Anal., 228 (2005), 207-233.

[38] R.E. Kalman, P. Falb, M. Arbib. Topics in mathematical system theory. McGraw-Hill, NY, 1969.

[39] A. Kasman, M. Gekhtman. Solitons and almost-intertwining matrices. J. Math. Phys., 42 (2001), 3540-3551.

[40] V.E. Katsnelson. Right and left joint system representation of a rational matrix function in general position. In: Operator Theory: Adv. Appl., 123 (2001), 337-400.

[41] B.G. Konopelchenko, C. Rogers. Bäcklund and reciprocal transformations: gauge connections. In: Nonlinear equations in applied sciences (W.F. Ames, C. Rogers, eds.), Academic Press, San Diego, 1992, 317-362.

[42] V.B. Kuznetsov, M. Salerno, E.K. Sklyanin. Quantum Bäcklund transformation for the integrable DST model. J. Phys. A, 33 (2000), No. 1, 171-189.

[43] D. Levi, O. Ragnisco, A. Sym. Dressing method vs. classical Darboux transformation. Nuovo Cimento B, 83 (1984), 34-41.

[LR] P. Lancaster, L. Rodman, Algebraic Riccati equations. Clarendon Press, Oxford, 1995.

[44] Q.P. Liu, M. Manas. Vectorial Darboux transformations for the Kadomtsev-Petviashvili hierarchy. J. Nonlinear Sci., 9 (1999), No. 2, 213-232.

[45] V.A. Marchenko. Nonlinear equations and operator algebras. Reidel Publishing Co., Dordrecht, 1988.

[46] V.B. Matveev. Positons: slowly decaying soliton analogs. Teoret. Mat. Fiz., 131 (2002), No. $1,44-61$.

[47] V.B. Matveev, M.A. Salle. Darboux transformations and solitons. Springer Verlag, Berlin, 1991. 
[48] R. Mennicken, A.L. Sakhnovich, C. Tretter. Direct and inverse spectral problem for a system of differential equations depending rationally on the spectral parameter. Duke Math. J., 109 (2001), No. 3, 413-449.

[49] R. Miura (ed.). Bäcklund Transformations. Lecture Notes in Math., 515, Springer-Verlag, Berlin, 1976.

[50] K. Pohlmeyer. Integrable Hamiltonian systems and interactions through quadratic constraints. Comm. Math. Phys., 46 (1976), No. 3, 207-221.

[51] C. Rogers, W.K. Schief. Bäcklund and Darboux transformations. Geometry and modern applications in soliton theory. Cambridge Texts in Applied Mathematics, Cambridge University Press, Cambridge, 2002.

[52] D.S. Sattinger, V.D. Zurkowski. Gauge theory of Bäcklund transformations II. Phys. D, 26 (1987), 225-250.

[53] A.L. Sakhnovich. Nonlinear Schrödinger equation on a semi-axis and an inverse problem associated with it. Ukr. Math. J., 42 (1990), No. 3, 316-323.

[54] A.L. Sakhnovich. The Goursat problem for the sine-Gordon equation and the inverse spectral problem. Russ. Math. Iz. VUZ, 36 (1992), No. 11, 42-52.

[55] A.L. Sakhnovich. Exact solutions of nonlinear equations and the method of operator identities. Lin. Alg. Appl., 182 (1993), 109-126.

[56] A.L. Sakhnovich. Dressing procedure for solutions of nonlinear equations and the method of operator identities. Inverse Problems, 10 (1994), 699-710.

[57] A.L. Sakhnovich. Iterated Darboux transform (the case of rational dependence on the spectral parameter). Dokl. Natz. Akad. Nauk Ukrain., 7 (1995), 24-27.

[58] A.L. Sakhnovich. Iterated Bäcklund-Darboux transformation and transfer matrix-function (nonisospectral case). Chaos, Solitons and Fractals, 7 (1996), 1251-1259.

[59] A.L. Sakhnovich. Iterated Bäcklund-Darboux transform for canonical systems. J. Functional Anal., 144 (1997), 359-370.

[60] A.L. Sakhnovich. Inverse spectral problem related to the $N$-wave equation. In: Operator Theory: Adv. Appl., 117, M.G. Krein volume (2000), 323-338.

[61] A.L. Sakhnovich. Generalized Bäcklund-Darboux transformation: spectral properties and nonlinear equations. JMAA, 262 (2001), 274-306.

[62] A.L. Sakhnovich. Dirac type and canonical systems: spectral and Weyl-Titchmarsh fuctions, direct and inverse problems. Inverse Problems, 18 (2002), 331-348. 
[63] A.L. Sakhnovich. Dirac type system on the axis: explicit formulas for matrix potentials with singularities and soliton-positon interactions. Inverse Problems, 19 (2003), 845-854.

[64] A.L. Sakhnovich. Non-Hermitian matrix Schrödinger equation: Bäcklund-Darboux transformation, Weyl functions, and $\mathcal{P} \mathcal{T}$ symmetry. J. Phys. A, 36 (2003), 7789-7802.

[65] A.L. Sakhnovich. Matrix Kadomtsev-Petviashvili equation: matrix identities and explicit non-singular solutions. J. Phys. A, 36 (2003), 5023-5033.

[66] A.L. Sakhnovich. Second harmonic generation: Goursat problem on the semi-strip, Weyl functions and explicit solutions. Inverse Problems 21 (2005), No. 2, 703-716.

[67] A.L. Sakhnovich. Non-self-adjoint Dirac-type systems and related nonlinear equations: wave functions, solutions, and explicit formulas. IEOT, 55 (2006), 127-143.

[68] A.L. Sakhnovich. Harmonic maps, Bäcklund-Darboux transformations and "line solution" analogues. J. Phys. A: Math. Gen., 39 (2006), 15379-15390.

[69] A.L. Sakhnovich. Skew-self-adjoint discrete and continuous Dirac-type systems: inverse problems and Borg-Marchenko theorems. Inverse Problems, 22 (2006), 2083-2101.

[70] A.L. Sakhnovich. Bäcklund-Darboux transformation for non-isospectral canonical system and Riemann-Hilbert problem. Symmetry Integrability Geom. Methods Appl., 3 (2007), 054, 11 pages.

[71] A.L. Sakhnovich. Discrete canonical system and non-Abelian Toda lattice: BäcklundDarboux transformation and Weyl functions. Math. Nachr., 280 (2007), No. 5-6, 1-23.

[72] A.L. Sakhnovich. Weyl functions, inverse problem and special solutions for the system auxiliary to the nonlinear optics equation. Inverse Problems, 24 (2008), 025026.

[73] A.L. Sakhnovich. Nonisospectral integrable nonlinear equations with external potentials and their GBDT solutions. J. Phys. A: Math. Theor., 41 (2008), 155204.

[74] A.L. Sakhnovich. Weyl functions, inverse problem and special solutions for the system auxiliary to the nonlinear optics equation. Inverse Problems, 24 (2008), 025026.

[75] A.L. Sakhnovich, J.P. Zubelli. Bundle bispectrality for matrix differential equations. IEOT, 41 (2001), 472-496.

[76] L.A. Sakhnovich. On the factorization of the transfer matrix function. Sov. Math. Dokl., 17 (1976), 203-207.

[77] L.A. Sakhnovich. Spectral theory of canonical differential systems, method of operator identities. Operator Theory: Adv. Appl., 107, Birkhäuser Verlag, Basel-Boston, 1999.

[78] C. Schiebold. Explicit solution formulas for the matrix-KP. Glasg. Math. J., 51A (2009), $147-155$. 
[79] C.L. Terng, K. Uhlenbeck. Bäcklund transformations and loop group actions. Commun. Pure Appl. Math., 53 (2000), 1-75.

[80] G. Teschl. Deforming the point spectra of one-dimensional Dirac operators. Proc. Amer. Math. Soc., 126 (1998), No. 10, 2873-2881.

[81] O.C. Wright, M.G. Forest. On the Bäcklund-gauge transformation and homoclinic orbits of a coupled nonlinear Schrödinger system. Physica D, 141 (2000), 104-116.

[82] A.E. Yagle, B.C. Levy. The Schur algorithm and its applications. Acta Appl.Math., 3 (1985), $255-284$.

[83] V.E. Zakharov, S.V. Manakov. Theory of resonance interaction of wave packages in nonlinear medium. JETP, 69 (1975), No. 5, 1654-1673.

[84] V.E. Zakharov, A.V. Mikhailov. Relativistically invariant two-dimensional models of field theory which are integrable by means of the inverse scattering problem method (Russian). Soviet Phys. JETP, 74 (1978), No. 6, 1953-1973.

[85] V.E. Zakharov, A.V. Mikhailov. On the integrability of classical spinor models in twodimensional space-time. Comm. Math. Phys., 74 (1980), 21-40.

[86] V.E. Zaharov, A.B. Shabat. On soliton interaction in stable media. JETP, 64 (1973), 16271639. 